\title{
Electron-anode interactions in particle-in-cell simulations of applied-B ion diodes
}

\author{
R. A. Vesey, T. D. Pointon, M. E. Cuneo, T. A. Mehlhorn, J. E. Bailey, D. J. Johnson, \\ T. J. Renk, and W. A. Stygar \\ Sandia National Laboratories, Albuquerque, NM 87185

\section{Abstract}

Particle-in-cell simulations of applied-B ion diodes using the QUTCKSILVER code [T. D. Pointon and M. P. Desjarlais, J. Appl. Phys. 80, 2079 (1996)] have been augmented with Monte Carlo calculations of electron-anode interactions (reflection and energy deposition). Extraction diode simulations demonstrate a link between the instability evolution and increased electron loss and anode heating. Simulations of radial and extraction ion diodes show spatial non-uniformity in the predicted electron loss profile leading to hot spots on the anode that rapidly exceed the $350-450^{\circ} \mathrm{C}$ range, known to be sufficient for plasma formation on electron-bombarded surfaces. Thermal desorption calculations indicate complete desorption of contaminants with $15-20 \mathrm{kcal} / \mathrm{mole}$ binding energies in high-dose regions of the anode during the power pulse. Comparisons of parasitic ion emission simulations and experiment show agreement in some aspects; but also highlight the need for better ion source, plasma, and neutral gas models.

PACS numbers: 52.58.Ei, 52.75.Pv, 52.25.Wz, 52.40.Hf

Corresponding author's e-mail address: ravesey@sandia.gov 


\section{REVISION 2}

\section{INTRODUCTION}

In order to deliver a shaped pulse of energy to an indirectly-driven high-yield (200-1000 MJ) inertial confinement fusion (ICF) capsule, a light ion driver must provide a sufficiently pure beam of ions with the desired range, with low divergence that allows the beam to be focused on target. Ref. 1 gives a recent review of progress toward this goal including specific driver requirements, beam transport schemes, and beam divergence requirements. Because the beam intensity on target scales as $P /(\Delta \theta)^{2}$, where $P$ is the beam power and $\Delta \theta$ is the divergence, efforts to reduce beam divergence have high leverage in improving beam intensity, and have received most of the experimental and theoretical attention. The overall beam divergence is composed of contributions from the ion source itself (nonuniform emission and transverse momentum sources associated with the ion emission process) and from electromagnetic instabilities that develop in the diode. As will be described in this paper, the electromagnetic instabilities that generate ion divergence are related to another chronic problem of high-power lithium ion diodes: the limitation of available lithium power due to the production of "parasitic" (non-lithium) ions from anode surface and bulk contamination [2,3]. An important result of this work is to quantify the direct relationship, in simulations, between the dominant electromagnetic instability and the level of electron leakage current. Electron loss is a serious concern for ion diodes because of energy deposition in anode materials and the subsequent formation of surface plasmas which then provide space-charge-limited sources of contaminant ions. Contaminant ions (non-lithium ions in the context of this paper) typically comprise a fraction of the beam population; on present diode experiments the fraction may be small for the first $10-20 \mathrm{~ns}$ of the beam pulse but later the beam is contaminant-dominated as the lithium current shuts off $[2,4]$. This paper describes the first efforts to include electron-anode interactions (reflection and energy deposition), anode heating, contaminant desorption, and the resulting parasitic ion emission in three-dimensional particle-incell simulations of ion diodes.

The simulations described here concern two different ion diode configurations, both 


\section{DISCLAIMER}

This report was prepared as an account of work sponsored by an agency of the United States Government. Neither the United States Government nor any agency thereof, nor any of their employees, make any warranty, express or implied, or assumes any legal liability or responsibility for the accuracy, completeness, or usefulness of any information, apparatus, product, or process disclosed, or represents that its use would not infringe privately owned rights. Reference herein to any specific commercial product, process, or service by trade name, trademark, manufacturer, or otherwise does not necessarily constitute or imply its endorsement, recommendation, or favoring by the United States Government or any agency thereof. The views and opinions of authors expressed herein do not necessarily state or reflect those of the United States Government or any agency thereof. 


\section{DISCLAIMER}

Portions of this document may be illegible in electronic image products. Images are produced from the best available original document. 
operated at Sandia National Laboratories: the SABRE extraction geometry and the PBFA II radial (or "barrel") geometry (Figures 1 (a-b)). In the SABRE geometry, an axial applied electric field $\left(\mathrm{E}_{\mathrm{z}}\right)$ accelerates an annular beam of ions suitable for extraction, post-acceleration, and transport, while a predominantly radial applied magnetic field $\left(B_{r}\right)$ insulates the anode from excessive electron flow across the gap. In the PBFA II geometry, a radial applied electric field $\left(\mathrm{E}_{\mathrm{r}}\right)$ accelerates ions toward the axis, while a vertical applied magnetic field $\left(B_{z}\right)$ provides insulation. Magnetic insulation is typically characterized by a quantity known as " $V_{\text {crit }}$ ", a critical voltage above which an electron emitted by the cathode crosses the anode-cathode (A-K) gap in one Larmor orbit. Assuming conservation of energy and $(\mathbf{E} \times \mathbf{B})$ drift canonical momentum for the electrons, electron leakage is prevented if the diode is operated at voltages less than $\mathrm{V}_{\text {crit }}$, which in cylindrically-symmetric systems is given by [6]:

$$
\frac{e V_{c r i t}}{m c^{2}}=\left[1+\left(\frac{e \Delta \Psi}{m c r_{a}}\right)^{2}\right]^{1 / 2}-1
$$

where $\Delta \Psi(r)=\Psi\left(r_{a}\right)-\Psi\left(r_{c}\right)$ is the difference in the magnetic stream function between the anode and cathode $\left(\Psi(r)=\mathrm{rA}_{\phi}\right.$, where $\mathrm{A}_{\phi}$ is the azimuthal component of the vector magnetic potential), $r_{a}$ is the anode radius, $e / m$ is the electron charge/rest mass ratio, and $c$ is the speed of light. Applied-B ion diodes thus employ a virtual cathode, a sheath populated by magneticallyconfined electrons emitted from cathode conductor surfaces, which defines the (dynamic) acceleration gap for the ions. The analytic diode theory of Desjarlais $[7,8]$ self-consistently models the diamagnetic motion of the azimuthally-drifting electron sheath, describing the nonlinear impedance characteristic and steady-state operating point of the diode. This theory explains the observed ion current enhancements for diode voltages much lower than $\mathrm{V}_{\text {crit }}$, within the constraints of energy and momentum conservation. (Enhancement is defined as $\mathrm{J}_{\mathrm{i}} / \mathrm{J}_{\mathrm{CL}}$, where $\mathrm{J}_{\mathrm{i}}$ is the ion current density and $\mathrm{J}_{\mathrm{CL}}$ is the space-charge limited Child-Langmuir current density.) 
Azimuthal asymmetries induced by electromagnetic fluctuations or by diode nonuniformities break momentum conservation, allowing the electron sheath to spread and migrate across the gap, resulting in some electron leakage for voltages less than $\mathrm{V}_{\text {crit- }}$ These essentially three-dimensional phenomena have been studied using the QUICKSILVER electromagnetic, relativistic particle-in-cell (PIC) code [9], primarily focusing on the effect such instabilities have on the divergence of the ion beam. Simulations in Cartesian geometry with parameters similar to PBFA II identified two primary electromagnetic instabilities present in applied-B ion diodes $[10,11]$ : the diocotron instability, an interaction of two sheath surface waves driven by shear in the electron drift velocity [12], and the two-stream-like ion mode driven by interaction of the ion beam with the electron sheath. In general, simulations in Cartesian[10,11], radial[13], and extraction[14] diode geometries show that a time-dependent transition from the diocotron to the ion mode occurs in the diode as the ion beam current ramps up. The dominant diocotron frequency $\left(v \sim\right.$ several GHz) is high compared with the inverse of the ion transit time $\left(\tau_{i} \sim 1-2\right.$ ns), while the dominant ion mode is characterized by $v \tau_{i} \sim 1$. As discussed in Ref. 11 , the oscillating azimuthal electric field $\left(E_{\phi}(t) \sim \sin (2 \pi v t)\right)$ generated by diocotron and ion mode instabilities generates an azimuthal velocity spread in the accelerated ion beam that scales predominantly as $1 / \mathrm{v}$. Therefore, the ion mode is expected to induce much more divergence in the ion beam than does the diocotron, as seen in numerous simulations. An electromagnetic, relativistic analysis has been developed which describes the growth of a modified two-stream instability very similar to the ion modes observed in 3-D simulations, which arises if electrons are allowed to respond to perturbations that are parallel to the applied magnetic field [15]. Experimentally, ion current density fluctuations and energy-momentum correlations have been measured which support this picture of instability evolution $[16,13,17]$. Because of the unacceptably high ion divergence associated with the ion mode, concentrated simulation $[10,11$, $13]$ and experimental effort $[17,18]$ has been spent in finding methods of operating ion diodes which avoid the presence of the ion mode. Although a connection between the ion mode transition and increased electron loss appears in a figure published for earlier radial diode 
simulations [13], and suggested by radial diode experimental results [17], this paper will focus on this effect and other aspects of electron loss in both extraction and radial diode simulations.

Section II introduces the problem of electron-anode interactions in high-voltage diodes, and previous work on electron reflection, anode heating, and contaminant desorption leading to parasitic ion currents. Section III discusses the main results of the Integrated TIGER Series (ITS) Monte Carlo calculations and their relevance to the ion diode PIC simulations. Section IV presents results of QUICKSILVER simulations of the SABRE ion diode, showing the links among the ion mode transition, electron loss, anode heating, and contaminant desorption. Section $\mathrm{V}$ describes simulations of the PBFA II radial diode including a thermal desorption model for the parasitic load, showing improved agreement with experimental results, particularly later in the pulse when non-lithium ions dominate the ion beam population.

\section{CONSEQUENCES OF ELECTRON REFLECTION AND ENERGY DEPOSITION}

The issue of electron reflection from anodes has been raised previously in the context of high-voltage diodes. Pereira [19] estimated that electron reflection from high- $\mathrm{Z}$ anodes in electron diodes reduces the electron current density relative to Child-Langmuir by up to $25 \%$ for unipolar flow, while the bipolar flow case predicted ion current enhancements of up to two times. Mosher et al. [20] included multiple electron backscattering in one-dimensional Poisson equation solutions for bipolar diodes, finding large enhancements in electron and ion current densities, depending on the magnitude of the reflected particle and energy yields. Quintenz and Widner [21] combined the features of a two-dimensional PIC code and a collisional electron Monte Carlo code to study the effects of electron reflection on the broadening of the electron beam pinch in relativistic electron beam diodes. In the cases explored by the references cited above, the electrons reflected into the anode-cathode gap had a lifetime of sufficient length to alter the overall space charge in the diode and affect its gross operating characteristics.

Studies of electron reflection in PIC simulations of applied-B ion diodes were motivated 
by experimental observations and by the expected effect of electron reflection on energy deposition in anodes. Spectroscopic measurements on the PBFA II ion diode show that after the onset of ion current, the electric field gradient near the anode is approximately zero, consistent with the local electron and ion densities being nearly equal out to $\sim 6 \mathrm{~mm}$ from the anode [22]. However, previous QUICKSILVER simulations without electron reflection yield an electron density profile that peaks $2 \mathrm{~mm}$ from the anode, then sharply decreases such that $n_{e} \ll n_{i}$ near the anode surface [22]. Reflection also affects the deposition of electron kinetic energy in the anode, which constitutes an important material heating source in ion diodes. A one-dimensional treatment of the effect of electron reflection on the energy deposition versus depth profile, assuming local electron redeposition, has previously been calculated and used to estimate heating and contaminant desorption for the PBFA II and SABRE ion diodes $[3,4,5]$. The present 3-D PIC diode simulations including reflection offer the advantage of treating non-local effects in calculating the 3-D anode energy deposition profile, effects that are important if electrons striking one region of the anode surface are reflected and return to deposit energy in another region of the anode. These simulations also model any direct effect that the altered electron density distribution may have on the electromagnetic instabilities.

The experiments considered in this paper used thin-film lithium fluoride $(\mathrm{LiF})$ as the ion source. Experimental [22] and theoretical [23] arguments suggest that LiF typically operates as a field-threshold source of ions. However, electron bombardment heats the anode ( $\mathrm{LiF}$ and substrate) materials, driving off resident contaminants that are then ionized by the primary and secondary electrons, producing near-surface plasmas. Spectroscopic evidence of contaminant anode plasmas is provided by measurements of visible and VUV emission from high charge states of carbon and oxygen [4]. On the PBFA II diode, the simultaneous observation of Stark-shifted and unshifted components of $\mathrm{Ba}$ II emission $\left(\mathrm{BaF}_{2}\right.$ co-evaporated with the $\mathrm{LiF}$ source film) indicated that parts of the $\mathrm{LiF}$ source were behaving as a field-threshold lithium emitter while other regions had formed surface plasmas [24]. Earlier work in low-impedance electron diodes 
REVISION 2

correlated the formation of anode plasmas with the specific heat of the anode material [25], suggesting thermal desorption as the mechanism for contaminant vapor/plasma formation. Sanford et al. [26] further quantified this correlation by measuring electron energy deposition in tantalum, titanium, and carbon anodes and found that surface temperatures of $350^{\circ}-450^{\circ} \mathrm{C}$ are sufficient for anode plasma formation.

Temperature-programmed desorption [27] studies of actual anode materials used in ion diodes are just beginning. However, data compiled from the literature provide guidance as to the ranges of contaminant binding energies to be expected, given the anode surface treatment and cleaning protocols used. These data are described in detail by Cuneo et al. [see Ref. 3, and references therein], who found that for stainless steel, typical binding energies are $14 \mathrm{kcal} / \mathrm{mole}$ for physisorbed $\mathrm{H}_{2} \mathrm{O}, 20-30 \mathrm{kcal} / \mathrm{mole}$ for chemisorbed hydrocarbons, and 10-40 kcal/mole for $\mathrm{H}_{2}$ on various metals. Estimates of the SABRE experimental anode temperature and associated thermal desorption show that as much as half of the $15 \mathrm{kcal} / \mathrm{mole}$ contaminant inventory may desorb before the time of peak lithium current [3]. Furthermore, SABRE data on electrode cleaning and substrate coatings show that the LiF films are permeable enough to allow the desorption of substrate contaminants unaffected by surface cleaning techniques [4]. These data are consistent with a thermal desorption model [3] which predicts that contaminant beam production should decrease with: (a) a reduction in electron loss, (b) higher material specific heat, (c) higher contaminant binding energies, and (d) smaller initial contaminant inventory. Results from these experiments and the temperature rise estimates motivated the implementation of anode temperature diagnostics in QUICKSILVER and are used in the development of the QUICKSILVER parasitic ion emission model described in Section III.D.

\section{INCLUSION OF ELECTRON-ANODE INTERACTIONS IN QUICKSILVER}

\section{A. INCIDENT ELECTRON FLUX}


QUICKSILVER simulations of ion diodes predict that the electrons transported across the anode-cathode gap strike the anode surface at fairly high incidence angles with respect to the surface normal. In the SABRE simulations, electrons emitted from the cathode tip dominate the leakage, which escalates dramatically after the transition from the diocotron mode to the ion mode. In contrast, the PBFA II simulations predict a significant flux of "feed" electrons, electrons emitted outside the diode which reach the anode surface throughout the power pulse due to the conductor geometry and applied magnetic field contours. (These feed electrons are an important feature of the PBFA II simulations and will be seen to cause the bulk of the predicted anode heating.) In both simulations, the average predicted incidence angle is approximately $80^{\circ}$ with a standard deviation of $\sim 5^{\circ}$. In all cases, the average energy of electrons striking the anode surface is a significant fraction ( $\sim 60-70 \%)$ of the diode voltage, translating to $\sim 3.5 \mathrm{MeV}$ for SABRE and $\sim 8 \mathrm{MeV}$ for PBFA II. For electrons at these high energies, bremsstrahlung production, Compton scattering, and photoelectric effects throughout the entire slowing-down process must be correctly modeled to provide an accurate description of energy deposition, electron escape, and photon escape. The Integrated TIGER Series (ITS) of coupled electron-photon Monte Carlo transport codes [28] provided the details of electron-material interactions for this work. While ITS models photon transport microscopically, electrons are treated using a condensed random walk model due to the large number of Coulomb collisions made during the slowing-down process. The ITS codes are ideal for this application because their capabilities include multidimensional, multimaterial transport of electrons and photons in the $1 \mathrm{keV}$ to $1 \mathrm{GeV}$ energy range. For the purposes of this work, two calculated quantities are of primary interest: (a) the probability distribution of electrons escaping the anode, and (b) the energy deposition profile in the anode material(s).

\section{B. ELECTRON REFLECTION}

For a one-dimensional treatment of electron reflection from a smooth surface, the essential task for the ITS simulations is to determine the probability distribution of reflected electrons as a 
function of the incident energy and polar angle, $\mathrm{E}$ and $\theta$, and the exit energy and polar angle, $\mathrm{E}^{\prime}$ and $\theta^{\prime}$. The dependence of the total reflected electron yield, $R$, (normalized to one source electron) is plotted in Figure 2 as a function of the incident electron energy and angle. Electron reflection is favored at grazing incidence regardless of incident energy due to a high probability of primary scattering ( $70-80 \%)$ and a significant probability of knock-on escape $(\sim 5-20 \%)$. As the angle of incidence decreases (becomes closer to normal incidence), the reflection probability generally decreases because the normal depth of penetration increases. Figure 2 shows that electrons incident at $\sim 80^{\circ}$ predicted by the QUICKSILVER simulations yield a $70 \%$ overall reflection probability.

Figure 3 shows the energy-angle distribution of reflected electrons, $P\left(E^{\prime}, \theta^{\prime}\right) d E^{\prime} d \Omega$ (where $d E^{\prime}$ and $d \Omega$ are the dimensions of uniform bins in energy and solid angle, respectively), calculated by ITS for a $4 \mathrm{MeV}$ primary electron incident on stainless steel at $80^{\circ}$ to normal. Two features are easily distinguished in this example: the backscattering peak due to nearly elastically scattered primaries, and the low-energy secondary electron population (knock-on and photon-produced electrons) described by a cosine distribution in exit angle. Of the total reflection yield for this case (0.67), backscattered primaries contribute 0.55 while secondaries account for 0.12 .

To include the presence of reflected electrons, the treatment within QUICKSIVER of electrons striking anode conductor surfaces was modified. Whereas previous simulations simply removed electrons from the system at conductor surfaces, the present model samples from scattering distributions derived from a matrix of ITS simulations. Following the methods used in Ref. 29, the problem of sampling from $\mathrm{P}\left(\mathrm{E}^{\prime}, \theta^{\prime}\right)$ is reduced to sampling from two one-dimensional 
distributions $\mathrm{f}_{1}$ and $\mathrm{f}_{2}$ given by:

$$
\begin{gathered}
f_{1}\left(E^{\prime}\right)=2 \pi \int_{0}^{\pi} P\left(E^{\prime}, \theta^{\prime}\right) \sin \theta^{\prime} d \theta^{\prime} \\
f_{2}\left(E_{o}^{\prime}, \theta^{\prime}\right)=P\left(E_{o}^{\prime}, \theta^{\prime}\right)
\end{gathered}
$$

Here, $f_{1}$ is the reflected electron distribution in energy (integrated over solid angle) and $f_{2}$ is the conditional one-dimensional distribution in angle for a given reflected energy $\mathrm{E}_{\mathrm{o}}$. The azimuthal angle dependence has been neglected, as evidenced by the factor of $2 \pi$ in the expression for $f_{1}$. The inverse cumulative distributions of $f_{1}$ and $f_{2}$ are calculated, and the actual quantities relevant for use within QUICKSILVER are the values of $E_{o}{ }^{\prime}$ and $\theta^{\prime}\left(E_{o}{ }^{\prime}\right)$ (or the reflected cosine, $\mu^{\prime}=\cos \left(\theta^{\prime}\right)$ ) corresponding to a uniform spacing in inverse cumulative distribution. These values define a set of equally probable results from which a selection is made based on random numbers.

The tables include the results of ITS cases in a run matrix spanning primary electron energies of $0.5-12 \mathrm{MeV}$ and incidence angles of $0^{\circ}-89^{\circ}$ to normal. The data for each incident energy $E$ and angle $\theta$ consist of an overall reflection coefficient, $R(E, \theta)$, a one-dimensional table of reflected energies, $E_{0}^{\prime}(E, \theta, \xi)$, and a two-dimensional table of the reflected cosines, $\mu^{\prime}(E, \theta, \xi, \eta)$. The parameters $\xi$ and $\eta$ designate the inverse cumulative distribution grid dimension. These tables enable QUICKSILVER to sample from the full four-dimensional reflected electron distribution each time a simulation electron particle is "killed" (removed from the simulation) on an anode conductor surface. The QUICKSILVER reflection algorithm performs three interpolations for each incident electron with energy $E_{p}$, polar angle $\theta_{p}$, and azimuthal angle $\phi_{p}$. First, the charge weight of the reflected electron is determined by multiplying the weight of the killed electron by the interpolated value of the reflection coefficient, $R\left(E_{p}, \theta_{p}\right)$. The energy, $E_{p}{ }^{\prime}$, and direction cosine, $\mu_{p}{ }^{\prime}$, of the reflected electron are determined by computing two random numbers and performing 
REVISION 2

interpolations in the reflected energy and cosine tables, respectively. Finally, the reflected electron is assigned an azimuthal angle based on its energy, approaching specular reflection $\left(\Delta \phi_{p}^{\prime}=0\right)$ as $E^{\prime} \rightarrow E_{p}$ and diffuse reflection as $E^{\prime} \rightarrow 0$. The reflected electron particle is then introduced into the system with the newly calculated charge weight, energy, and momenta. This reflected electron may at some later time return to the anode surface where it becomes the primary electron in a subsequent reflection event.

\section{ELECTRON ENERGY DEPOSITION IN ANODE MATERIALS}

The same matrix of ITS runs described in the previous section was used to construct a table of energy deposition versus depth for use in conjunction with QUICKSILVER. In the cases considered here, a $1 \mu \mathrm{m}$ zone of $\mathrm{LiF}$ rests on a substrate of stainless steel 304 . Figure 4 shows the energy deposition versus depth near the anode surface for a primary electron impacting the surface with $E=4 \mathrm{MeV}$ at $\theta=80^{\circ}$ to normal. The anode heating results shown later in this paper assume an adiabatic temperature rise. The neglect of thermal conduction is justified by the length of the typical diode power pulse $(\sim 50 \mathrm{~ns})$, the thermal diffusion lengths for LiF and stainless steel $(\sim 1 \mu \mathrm{m}$ in $10 \mathrm{~ns})$, and the flat energy deposition profile pictured in Figure 4 which implies a weak temperature gradient in the stainless steel. The anode heating results will focus on the stainless steel substrate because the high-energy electrons interact more strongly with the stainless steel than with the lower atomic number LiF. Because stainless steel and LiF have similar volumetric heat capacities, the stainless steel temperature will rise faster than the LiF temperature for a given electron flux. Also, although adsorbed contaminant levels have been measured under diode conditions for $\mathrm{LiF}$ [30], experiments with substrate coatings indicate that bulk contaminants are also released as the substrate is heated $[3,4]$. Thus, for the purposes of anode heating and contaminant desorption calculations, the LiF layer will be essentially ignored.

Anode heating has been evaluated not only by postprocessing killed electron data saved from QUICKSILVER simulations, but also with an anode temperature tally internal to QUICKSILVER. 
As with the treatment of electron reflection, QUICKSILVER reads energy deposition data, tabulated as energy deposition per unit depth as a function of the incident electron energy and angle, $\mathrm{Q}(\mathrm{E}, \theta)$. The tables of $\mathrm{Q}(\mathrm{E}, \theta)$ for each 1- $\mu \mathrm{m}$ zone (depicted in Figure 4) are included in the data file, but typically the anode temperature tally is only concerned with the top $1 \mu \mathrm{m}$ of stainless steel substrate. For an electron killed in a given computational cell on the anode surface, the energy deposition $\mathrm{Q}\left(\mathrm{E}_{\mathrm{p}}, \theta_{\mathrm{p}}\right)(\mathrm{J} / \mathrm{cm})$ is interpolated from the table, the average volumetric heat source for the cell is calculated, and the local temperatures updated assuming adiabatic conditions. The updated local temperatures are therefore accessible for output by existing diagnostic routines or for use by other models such as the parasitic ion emission model described below.

\section{PARASITIC ION EMISSION MODEL}

A direct implementation within the QUICKSILVER code of the detailed physics of contaminant desorption, neutral gas expansion, ionization, charge exchange, and the dynamics of dense surface plasmas is infeasible in the near term. First, QUICKSILVER presently contains no neutral particle physics, so major code modifications would be needed to directly model the desorption, expansion, and interactions of neutrals with charged particle species. A second and more fundamental reason is that an explicit particle-in-cell simulation of the cold $\left(T_{e} \sim 5-10 \mathrm{eV}\right)$, dense $\left(n_{e}>10^{15}-10^{16} \mathrm{~cm}^{-3}\right)$ plasma layers expected near electrode surfaces for pulsed power applications [31] would require much higher spatial resolution (minimum $\Delta \mathrm{x}<0.2-0.7 \mu \mathrm{m}$ ) and much smaller time steps $\left(\Delta \mathrm{t} \sim \Delta \mathrm{x} / \mathrm{c}<6 \times 10^{-4}-2 \times 10^{-3} \mathrm{ps}\right)$ than present simulations, in order to resolve the plasma Debye length and to satisfy the field solver Courant condition. Present simulations of SABRE, 'modeling $1 / 4$ of the azimuthal extent with a minimum $\Delta x=0.4 \mathrm{~mm}$ and $\Delta t=1 \mathrm{ps}$, occupy 20 megawords of memory and require about 100 hours of CPU time on a Cray J90 [32]. Hybrid fluid-PIC codes such as IPROP [33] and DYNAID [34] are under development to model dense electrode plasmas in detail, but both codes operate in idealized geometries, and are 
REVISION 2

presently unable to include the full 3-D diode physics modeled by QUICKSILVER. However, through the use of results from these hybrid models together with diode experimental results, some macroscopic aspects of neutral contaminant desorption, expansion, and ionization can be included in QUICKSILVER.

The parasitic ion emission model presently employed by QUICKSILVER is based on the thermal desorption model for contaminant desorption and ionization detailed in Ref. 3 and summarized in Section II. It is based on the experimental support of the hypothesis that electron energy deposition raises the temperature of anode materials, driving the thermal desorption of resident impurities. These (initially neutral) contaminants are partially ionized close to the anode by primary and surface secondary electrons, and an ionization avalanche breaks down the neutral layer once its thickness is comparable to a plasma electron Larmor orbit diameter [5]. After this time, the contaminant plasma layer becomes a space-charge-limited source of parasitic ions and a sink for gap magnetic flux which possibly contributes to impedance collapse [8]. Because of the limitations described previously, the plasma layer contribution to impedance collapse cannot be directly modeled by QUICKSILVER, but the initial stage of parasitic ion emission overwhelming lithium emission may be approximated. A macroscopic treatment of the effects of these processes within QUTCKSILVER begins by processing the simulation electron particles which strike the anode, using the anode temperature tally described in the previous section. A unique feature offered by QUTCKSIVER is the fully 3-D calculation of electron transport under the influence of dynamically evolving instabilities that develop in the electron sheath, thereby treating growth, saturation, and nonlinear coupling of modes supported by the system. The effect of electron sheath instabilities on the field-threshold ion emission spatial profile has been discussed[13]; the parasitic ion emission model adds another degree of complexity by linking the onset of contaminant species emission to the calculated electron loss. By the same token, the introduction of contaminant ion current may then feed back on the electron sheath instabilities and electron loss characteristics.

Ion emission in QUICKSILVER is handled by creating simulation particles in cells directly 
adjacent to conductor surfaces [13]. With the addition of the parasitic ion emission model, QUICKSILVER now allows three different models for ion emission:

(1) Space-charge-limited (SCL) emission sets the emission threshold electric field, $\mathrm{E}_{\mathrm{thr}}=0$, modeling emission from a dense, electric field-excluding plasma with a thickness less than the cell thickness $\Delta \mathrm{x}_{\mathrm{n}}$.

(2) Field-threshold (FT) emission employs a user-defined non-zero value of $\mathrm{E}_{\mathrm{thr}}$ to model the behavior of $\mathrm{LiF}$ ion sources.

(3) Temperature-threshold space-charge-limited (TSCL) emission delays the onset of SCL emission within a cell until a user-defined anode temperature threshold has been exceeded.

For the TSCL emission model, the user-defined temperature threshold, $\mathrm{T}_{\text {thr }}$ and an initial delay time, $\tau_{\mathrm{d} 0}$, determine the time at which a cell begins SCL emission of a prescribed ion species. The delay time is included to account for the details of contaminant desorption and ionization not being directly modeled by QUICKSILVER. In general, the time at which a TSCL cell will begin $\mathrm{SCL}$ emission is given by:

$$
t_{e m}=t_{t h r}+\tau_{d 0}+\frac{\partial \tau_{d}}{\partial T}(T) \Delta T
$$

where $t_{\text {thr }}$ is the time at which the temperature, averaged over the computational cell, exceeds $T_{t h r}$ A functional dependence of the delay time on the anode temperature may be specified to model the acceleration of the desorption and ionization processes by a rapidly rising anode temperature. For the parasitic load simulations described in later sections, a fixed temperature threshold, $\mathrm{T}_{\mathrm{thr}}=250^{\circ} \mathrm{C}$, and a fixed delay time, $\tau_{\mathrm{d} 0}=5 \mathrm{~ns}$, have been used. A temperature threshold lower than the experimentally-measured $350-450^{\circ} \mathrm{C}$ range [26] was used to compensate for the inherent temperature averaging effect of the $2-3 \mathrm{~mm}$ computational cell size. (The exact impact position of each electron on the anode is known and may be used by postprocessing routines to provide highresolution temperature maps such as those in Section IV.C. However, the QUICKSILVER 
internal temperature tally used by the TSCL model relies on the spatial resolution provided by the coarser computational mesh, which leads to artificial smoothing of the computed temperature profile.) The delay time of $5 \mathrm{~ns}$ is consistent with the contaminant layer ionization predictions of Welch et al. [5,3].

A key new feature of the parasitic ion emission model is the ability to overlap FT emission regions with TSCL emission regions. For such overlap regions, the simulation begins with all FT emission cells on and all TSCL emission cells off. Once a particular TSCL cell reaches its emission time, $\mathrm{t}_{\mathrm{em}}$, FT emission is completely switched off, replaced by SCL emission. For a typical ion diode simulation, this means that cells which began the simulation as field-threshold lithium emitters may switch over to space-charge-limited emission of some contaminant ion (e.g. protons) if the parasitic ion emission model conditions are met. In this model, spatially nonuniform electron deposition and anode heating will lead to equally non-uniform patches of contaminant ion emission interspersed with lithium-emitting regions, which is consistent with the non-uniformity seen in experimental spectroscopic measurements[22]. However, due to the exclusive FT-or-SCL ion emission model, time-dependent mixed-species emission from partially ionized anode plasmas is not modeled properly.

\section{SIMULATIONS OF THE SABRE EXTRACTION ION DIODE}

\section{A. SIMULATION SETUP}

An azimuthal $(\mathrm{R}-\mathrm{z})$ slice of the SABRE simulation geometry is shown in Figure 5, with streamlines of the applied magnetic field generated by the ATHETA code [35]. $V_{\text {crit }}$ varies from 10.2 MV to 8.1 MV from the inner to outer radii of the anode face. The conductor geometry of SABRE is nominally axisymmetric and is treated as such in the simulations presented here. The actual SABRE cathode conductor does not extend toward the anode at small radius; this artificial feature is added because the current version of QUICKSILVER is unable to zone down to $R=0$ in 
cylindrical coordinates. This treatment, which involves extending the cathode back and imposing a mirror boundary condition on the inner coaxial line, has been tested against simulations with TWOQUICK (a two-dimensional code which zones to $\mathrm{R}=0$ ) [35]. These tests, along with QUICKSILVER tests using alternate boundary treatments, suggest that the overall diode behavior is insensitive to the details of this boundary, although electron trajectories very near this boundary are certainly suspect. The 3-D model of the outer coaxial feed shown in Figure 5 extends back to $\mathrm{Z}=-30 \mathrm{~cm}$, at which point is connected a one-dimensional $28 \mathrm{~ns}, 40 \Omega$ vacuum transmission line model. The transmission line is driven by an open-circuit waveform with a $38 \Omega$ source impedance, to model the SABRE ten-cavity linear induction voltage adder.

The computational constraints governing the simulation setup are similar to those described for simulations of the PBFA II radial diode [13]. To resolve the 1-cm anode-cathode gap, a non-uniform mesh of 20 cells has been used, with $\Delta z=0.4 \mathrm{~mm}$ at the anode. Based on the Courant condition for the smallest cell, the time step is set to $1 \mathrm{ps}$. The uniform azimuthal mesh spacing is typically $\Delta \phi=\pi / 64$, with either 16 or 32 azimuthal cells, yielding $1 / 8$ or $1 / 4$ diode simulations. Periodic boundary conditions are applied at the two azimuthal boundaries of the system, thus limiting the available azimuthal modes to multiples of $m=8$ and $m=4$ for the $1 / 8$ and $1 / 4$ diode simulations, respectively. The use of these reduced azimuthal sections is justified by an additional simulation, performed on a coarser mesh in simplified geometry for efficiency, which modeled the full $2 \pi$ azimuthal extent. Focusing on the ion mode phase of the simulation, the dominant modes for the $2 \pi$ simulation, in order of decreasing oscillating electric field magnitude, are $\mathrm{m}=8,14,10,16,9$, and 11 , with the $\mathrm{m}=8$ having more than 1.5 times greater magnitude than any other mode. Furthermore, the contributions of the $m=14$ and $m=10$ modes appear to be transitory, peaking as the diocotron modes are overwhelmed by lower frequency ion modes. Therefore, although the $1 / 8$ and $1 / 4$ diode simulations model only the $m=8$ and $m=16$ modes from this set, they do at least capture the most virulent ion mode and its harmonics, which will be seen to be important for both ion beam divergence and electron loss physics. 


\section{REVISION 2}

Four species of electrons are used for diagnostic purposes in these simulations, composed of two primary and two reflected species. Of the primary species, "feed" electrons are emitted from the portion of the cathode between $Z=-15 \mathrm{~cm}$ and the outer radius of the base of the cathode tip. "Diode" electrons are emitted from the remaining cathode structure, including the cathode tip and inner cathode coil housing. For each of these two primary species, a reflected electron species is created by the scattering of primaries after they impact the anode face. The anode face is defined as the flat annular region, $4.22<\mathrm{R}<6.68 \mathrm{~cm}$, located at $\mathrm{Z}=0$ in Figure 5. Of this, the region defined by $4.43<\mathrm{R}<6.49 \mathrm{~cm}$ is defined as lithium-emitting. Lithium emission is treated using the field-threshold model [13] with a threshold electric field of $E_{t h r}=6 \mathrm{MV} / \mathrm{cm}$. Lithium particles killed at the gas cell boundary are then saved for analysis of ion beam profiles and divergence.

\section{B. ELECTRON REFLECTION RESULTS}

Despite the $70 \%$ total reflection probability for the "average" primary electron noted in Section III.B, very little direct effect of reflected electrons on the gross diode behavior is seen in QUICKSILVER simulations. The slight differences in quantities such as the diode voltage, ion beam current, and ion beam divergence are no larger than variations due to changing the random number seed. Particle diagnostics reveal that on average, reflected electrons spend $\sim 0.1 \mathrm{~ns}$ in the system, while primary feed electrons spend $\sim 4 \mathrm{~ns}$ and primary diode electrons spend $\sim 18 \mathrm{~ns}$ in the system prior to being killed. Thus it appears that reflected electrons are unable to build up sufficient space charge in the diode gap to affect gross characteristics. This is born out by Figure 6 , showing the azimuthally-averaged profile of electron densities across the anode-cathode gap, summing the feed and diode species contributions. Only at the anode surface $(Z=0.0)$ are the two populations at all comparable, with the primary electron density $\sim 3$ times higher than the reflected electron density. Therefore, the addition of electron reflection alone does not help to explain the apparent discrepancy between the simulation and the spectroscopically derived charge densities [22] noted in Section II. Nonetheless, the process of electron reflection from the anode 
is undoubtedly taking place in experimental diodes, and is routinely included in subsequent simulations. Three expected effects of electron reflection are: (a) altered anode heating spatial profiles due to electron energy redistribution, (b) enhanced anode surface heating due to shorterrange recycled electrons, and (c) increased near-anode neutral layer ionization rates due to the surface secondary electron cascade.

The reflection process obviously increases the magnitude of the flux of electrons through the anode surface. Figure 7 shows a snapshot of the azimuthally-integrated radial profile of incident electron flux (normalized to the peak primary flux) for the two groups at $t=74 \mathrm{~ns}$, well into the ion mode phase. The peak primary electron deposition occurs near the inner radius of the anode face, while the reflected electron deposition makes its largest contribution relative to the primary electron deposition at larger radii, due to the positive average radial component of the primary electron velocity which is preserved after scattering. This spatial profile is important because the lithium-emitting portion of the anode face is restricted to $4.43<\mathrm{R}<6.49 \mathrm{~cm}$. Figure 8 displays the primary and reflected electron currents into the anode face for this simulation, showing the surprising result that the two currents are nearly identical, despite the energy and angle dependence of the reflection coefficient, and despite the different spatial loss profile for the two groups. Considering a single primary electron at $4 \mathrm{MeV}$ and $80^{\circ}$ incidence, the reflection coefficients are 0.68 and 0.36 for the first two generations of reflected electrons. The total return flux expected for the first two reflected generations is therefore $0.68+0.36=1.04$ times the primary flux. This result, combined with the charge weight cutoff used in the QUICKSILVER simulations which limits the number of reflected generations, and with the short average residence time of reflected electrons, explain the similarity of the time histories of the primary and reflected electron loss currents.

\section{ANODE HEATING RESULTS}

The main result of simulations focusing on SABRE anode heating is to quantify the link 


\section{REVISION 2}

between the transition from the diocotron mode to the ion mode and the onset of increased electron energy deposition leading to a rapid, spatially non-uniform anode temperature rise. The simultaneous appearance of the ion mode instability, high ion beam divergence, and increased electron loss was seen in earlier QUICKSILVER simulations [11]. PBFA II experiments show a time-correlation between the appearance of an ion beam energy-momentum correlation (indicative of the low frequency ion mode instability), an increase in the beam focal spot size indicating higher divergence, and the production of hydrocarbon beam components [17], although a cause-effect relationship is not established. Figures 9 (a-d) summarize the QUICKSILVER anode heating results for a 1/4 diode simulation of SABRE, with the anode temperature initialized at $20^{\circ} \mathrm{C}$. Figure $9($ a) is a time history of the $m=8$ mode from a spatial fast-Fourier transform of the azimuthal electric field, showing the evolution from the high frequency, low amplitude diocotron instability, through a transition period to the late time behavior dominated by a low frequency, high amplitude ion mode instability. Figure 9(b) is a history of the lithium ion microdivergence, showing 4-8 milliradians (mrad) in the diocotron phase and more than $40 \mathrm{mrad}$ once the ion mode dominates. Figure 9(c) shows that at the ion mode transition, the electron loss power delivered to the anode face rises from $\sim 10 \mathrm{GW}$ up to $\sim 150 \mathrm{GW}$, which for SABRE yields average energy fluxes of $\sim 2 \mathrm{GW} / \mathrm{cm}^{2}$. The consequences of this electron loss are shown in Figure 9(d), which shows the maximum temperatures overall, maximum temperatures for $\mathrm{R}>4.5 \mathrm{~cm}$ (in the lithium emission region), and an average temperature based on a uniform distribution of power over the anode face. This plot indicates the spatial non-uniformity of the electron flux, leading to peak temperatures 5 times higher than the average. The maximum temperature reaches the $350^{\circ}-450^{\circ} \mathrm{C}$ range relevant for surface plasma formation within a few nanoseconds of the ion mode transition. Although the entire 3-D anode conductor temperature field has been calculated based on the QUICKSILVER killed electron diagnostics, the most concentrated electron loss and heating occur on the anode face in this simulation, with relatively minor heating taking place in the outer coaxial feed region $\left(\mathrm{T}_{\max } \sim 150^{\circ} \mathrm{C}\right.$ by $\left.\mathrm{t}=78 \mathrm{~ns}\right)$. 
The electron flux and energy deposition into the anode face exhibit distinct radial and azimuthal structures that sweep azimuthally in time. The radial structure of the electron loss reflects the harmonic structure of the ion mode along the applied magnetic field [13], while the

azimuthal sweeping occurs at the ion mode phase velocity $\left(v_{\phi} \sim c / 22\right.$ in this case). Figures 10 (a-f) display snapshots of the volumetric heat source and temperature in the top $10 \mu \mathrm{m}$ of stainless steel, projected onto a $90^{\circ}$ sector of the anode face. The $\mathrm{E}_{\mathrm{z}} \times \mathrm{B}_{\mathrm{R}}$ drift direction and ion mode phase velocity are directed counter-clockwise in this figure. (Black corresponds to heat sources exceeding $400 \mathrm{GW} / \mathrm{cm}^{3}$ and temperatures exceeding $400^{\circ} \mathrm{C}$.) The first strong electron burst at $t=65$ ns deposits a significant amount of energy near the location on the anode face seen in Figure 10 (a). Since the anode temperature is proportional to the integrated energy deposition, this particular spot gets an initial boost in temperature and remains the hottest spot as the simulation progresses, even though the energy deposition pattern sweeps azimuthally. The heat source snapshots in Figures 10(c) and (e) clearly show the $m=8$ structure of the dominant ion mode driving the electron loss. By $t=75$ ns (Figure 10(f)), significant areas of the anode exceed the $350^{\circ}-450^{\circ} \mathrm{C}$ range important for anode plasma formation. An important effect of reflected electrons in this simulation is to raise the temperature in the lithium-emitting region by about $50 \%$ relative to primaries alone, as alluded to in Section III.C, referring to Figure 7. Another conclusion of this simulation is that if the electron loss could be maintained at the level seen in the diocotron phase, the maximum temperature would not reach $300^{\circ} \mathrm{C}$ during the power pulse, and little or no surface plasma formation due to thermal processes would be expected.

\section{CONTAMINANT DESORPTION ESTIMATES}

The rapid and spatially non-uniform heating of the anode surface will lead to desorption of contaminants at differing rates for different positions. Following earlier work [3], desorption is modeled by postprocessing QUICKSILVER killed electron diagnostics, by the following equation: 


$$
\frac{d n_{a}}{d t}=-\left(n_{a}^{x} v e^{-\frac{Q}{R T}}+n_{a} \sigma_{d e s} \Gamma_{e}\right)
$$

where $n_{a}$ is the adsorbate concentration $\left(\mathrm{cm}^{-2}\right), Q$ is the contaminant binding energy (kcal/mole), $v$ is the pre-exponential rate constant for the particular process considered $\left(\mathrm{cm}^{2(\mathrm{x}-1)} \mathrm{s}^{-1}\right), R$ is the gas constant $\left(1.987 \times 10^{-3} \mathrm{kcal} / \mathrm{mole} / \mathrm{K}\right), T$ is the local substrate temperature $(\mathrm{K}), \sigma_{\text {des }}$ is the electron-stimulated desorption cross-section $\left(\mathrm{cm}^{2}\right)$, and $\Gamma_{e}$ is the local electron flux through the surface $\left(\mathrm{cm}^{-2} \mathrm{~s}^{-1}\right)$. The exponent $\mathrm{x}$ represents the order of the particular desorption process, and in general $Q, v$, and $\sigma_{d e s}$ are functions of coverage, contaminant species, and binding state.

Experimental measurements of these fundamental quantities typically focus on pristine crystal planes with $<1$ monolayer coverage and so are not directly applicable to our problem, with its uncharacterized distribution of contaminants in varying bound states. Expected ranges of binding energies were discussed in Section II. Stimulated desorption cross-section measurements for high-energy electrons on practical materials are scarce in the literature. The limited values for stainless steel range from $5 \times 10^{-16} \mathrm{~cm}^{2}$ for $600 \mathrm{eV}$ electrons [36], to $5 \times 10^{-18}$ to $1 \times 10^{-16} \mathrm{~cm}^{2}$ for 1.4 $\mathrm{keV}$ electrons [37], to $10^{-17} \mathrm{~cm}^{2}$ for $100 \mathrm{keV}$ electrons [38].

Figure 11 shows time histories of the two QUICKSILVER-postprocessed desorption frequencies (the terms multiplying $n_{a}$ in equation 4), azimuthally averaged at $\mathrm{R}=4.3 \mathrm{~cm}$, for firstorder thermal desorption with $v=10^{13} \mathrm{~s}^{-1}, Q=15 \mathrm{kcal} / \mathrm{mole}$, and stimulated desorption characterized by $\sigma_{d e s}=5 \times 10^{-16} \mathrm{~cm}^{2}$. For the purposes of stimulated desorption the value of $\Gamma_{e}$ includes the primary and reflected electron fluxes. Processes with frequencies on the order of 0.1 $1 \mathrm{~ns}^{-1}$ are relevant for the pulse. Stimulated desorption is seen to rise for this cross-section only after the ion mode transition, but is dominated within $2-3 \mathrm{~ns}$ by the rapidly rising thermal desorption frequency which reaches $1-10 \mathrm{~ns}^{-1}$ during the pulse. (Similar arguments on the 
dominance of thermal desorption were presented in Ref. 3.)

Given the spatial non-uniformity in the anode temperature demonstrated in the previous section, the desorption rates are even more non-uniform due to the exponential dependence of thermal desorption. Figures 12(a-c) show the time history of the desorbed contaminant fraction for several desorption models. The desorbed fraction is just the initial contaminant inventory minus the integral of the right-hand-side of equation 2 normalized by the initial inventory. Figure 12(a) corresponds to the first-order, constant $Q(15 \mathrm{kcal} / \mathrm{mole})$ case mentioned above, separating out the contribution of stimulated desorption. Figure 12(b) corresponds to a first-order process with $Q$ varying linearly from $15-20 \mathrm{kcal} / \mathrm{mole}$ as the desorbed fraction increases from 0 to 1 , to model desorption from a distribution of binding energies typical of engineering surfaces. Figure 12(c) corresponds to a second-order thermal desorption process representative of hydrogen on stainless steel which appears to desorb as $\mathrm{H}_{2}$ after surface migration and recombination steps. Desorption data show a sensitivity to oxide layer thickness and surface treatment; curves are plotted for $v=4.33 \times 10^{-3} \mathrm{~cm}^{2} \mathrm{~s}^{-1}, Q=16.1 \mathrm{kcal} / \mathrm{mole}$, and $v=1.44 \times 10^{-4} \mathrm{~cm}^{2} \mathrm{~s}^{-1}, Q=18.7 \mathrm{kcal} /$ mole from data compiled by Langley[39]. Although the detailed shape of the desorption history depends on the model parameters, Figures 12(a-c) show that within the range thought to be relevant for SABRE, a large fraction of the contaminant inventory is desorbed from the highestdose areas during the beam pulse, within $10 \mathrm{~ns}$ of the ion mode transition which takes place at $\mathrm{t} \sim 65 \mathrm{~ns}$. Perhaps more importantly, at least $10 \%$ of the inventory in areas outside those receiving the highest electron doses are released, corresponding to many desorbed monolayers since the $\mathrm{LiF}$ hydrocarbon contaminant inventory has been measured at tens of monolayers [30], and the substrate stainless steel contaminant inventory is expected to be at least as high.

The SABRE anode heating and desorption results presented above demonstrate that, in this diode simulation geometry, the ion mode transition not only induces an unacceptably high divergence in the lithium beam, but also causes a dramatic increase in the electron loss to the anode face. This increased electron loss leads to rapid anode heating, which drives the desorption 


\section{REVISION 2}

of resident contaminants on nanosecond timescales. The results of this simulation suggest that efforts to delay or inhibit the ion mode transition (e.g. through the use of electron limiters $[40,10,11])$ to maintain a low divergence ion beam may also mitigate the problem of parasitic ion emission from the anode face. However, the same thermal processes described above will cause the limiter itself to become a parasitic ion source. Therefore, the limiter must be placed in a location far enough outside the accelerating gap so that small parasitic ion currents will be generated, but close enough to collect sufficient sheath electron current to inhibit the ion mode transition. The combined simulation capabilities of QUICKSILVER to model the 3-D diode physics and ITS to model the detailed electron-material interactions constitute a powerful tool to guide the design of such systems.

\section{E. PARASITIC ION EMISSION MODEL RESULTS}

The QUICKSILVER results presented in Sections IV.B-D included only lithium ion emission; in this section a simulation utilizing the anode temperature tally and parasitic ion emission model will be discussed. Initially, the lithium-emitting portion $(4.43<\mathrm{R}<6.49 \mathrm{~cm})$ of the anode face is prescribed to be a field-threshold (FT) $\mathrm{Li}^{+1}$ emitter with $\mathrm{E}_{\mathrm{thr}}=6 \mathrm{MV} / \mathrm{cm}$. In addition, the entire anode face $(4.22<R<6.68 \mathrm{~cm})$, is prescribed to be a temperature-threshold space-charge-limited (TSCL) emitter of protons, with $\mathrm{T}_{\mathrm{thr}}=250^{\circ} \mathrm{C}$ and $\tau_{\mathrm{d} 0}=5 \mathrm{~ns}$. Although other contaminant species are also observed experimentally, protons were chosen as the sole parasitic species for this simulation because they are the most damaging to the lithium efficiency.

Figures $13(\mathrm{a}-\mathrm{c})$ show the time histories of the diode voltage and particle currents for this parasitic load simulation compared to the baseline simulation of Sections IV.B-D. Significant differences are seen after $\mathrm{t}=70 \mathrm{~ns}$, the time at which the first emission of protons occurs. From this time onward, the field-threshold lithium source must compete with a space-charge-limited proton source which is growing in emission area due to continued electron heating. Figure 13 (b) shows the rapid decay of the lithium current as the parasitic proton current ramps up, while the 
diode voltage decreases as seen in Figure 13 (a) in response to the increasing total ion current. Figure 13 (c) shows a feedback effect on the electron loss current, due to the rapid localized switch from FT lithium emission to SCL proton emission in those areas exceeding the temperature threshold. As the proton current is ramping up, the global electron loss current is seen to drop to very low levels. This effect is due to localized decreases in electron loss to SCL proton emitting areas of the anode face. Figures $14(\mathrm{a}-\mathrm{f})$ represent head-on views of the simulated anode face at $\mathrm{t}=71.5,76$, and $80.5 \mathrm{~ns}$, showing contours of electron flux and the positions of the two species of ion particles within $1 \mathrm{~mm}$ of the emitting surface (ion velocity vectors are directed out of the page). Snapshots (a-b), at $t=71.5 \mathrm{~ns}$, show the electron flux distribution corresponding to the established lithium $\mathrm{m}=8$ ion mode, and the first emission of protons from the hottest spot on the inner radius of the anode face. The next snapshots ( $t=76 \mathrm{~ns})$ show a dramatic drop in the electron flux where the proton emission is highest, most likely due to the sudden change in the near-anode electric field accompanying the switch from field-threshold emission $\left(\mathrm{E}_{\mathrm{thr}}=6 \mathrm{MV} /\right.$ $\mathrm{cm})$ to space-charge-limited emission $\left(\mathrm{E}_{\mathrm{thr}}=0\right)$. By $\mathrm{t}=80.5 \mathrm{~ns}$, large areas of the anode face are emitting only protons, and significant electron loss occurs only in those regions in which FT lithium emission is still taking place. From $\mathrm{t}=80 \mathrm{~ns}$ on, the non-uniform emission of the two species clearly disrupts the previously-dominant $m=8$ lithium ion mode, and global electron loss is low. This is confirmed by the FFT diagnostics which show the lithium ion mode oscillations decaying in magnitude. This is expected to remain the case until enough of the anode face is a pure proton emitter such that a proton ion mode instability can dominate.

\section{F. COMPARISON OF SIMULATIONS WITH SABRE EXPERIMENTS}

Some useful comparisons between the parasitic load simulation results and observed SABRE ion diode behavior can now be made. For comparison with the presented QUICKSILVER results, SABRE shots with similar conductor and applied magnetic field configurations were selected (although substrate coatings varied). The location of various experimental diagnostics are indicated schematically in Figure 1(a). The dB/dt ("B-dot") monitors indicated as Bdot1 and 


\section{REVISION 2}

Bdot2 actually represent arrays of monitors located at six and four azimuthal locations, respectively. Bdot1 measures the total cathode current, while Bdot2 measures the total current flowing at radii smaller than the cathode tip radius. Filtered large (6-12 mm aperture) Faraday cups ("F-cups") provide current measurements for total ions, lithium, and protons by selective filtering. Taking differences of various global current measurements allows other interpretations to be made. For example, the difference Bdot1-Bdot2 is interpreted as the electron current emitted by the cathode tip ("Iektip"). Also, the difference of Bdot2 and the total ion current represents a current flowing at small radius ("Icenter"), and is interpreted as electrons emitted by conductor surfaces such as the inner cathode coil housing. Direct measurements of the electron flux striking the anode face are provided by an array of tungsten-collimated silicon p-i-n diodes ("Xrpins") viewing bremsstrahlung and line radiation emitted from the anode face. The Rutherford scattering beam magnetic spectrometer ("MSBeam") is referred to here primarily as a diode voltage diagnostic, giving the time-dependent average lithium ion energy.

A total of 25 selected shots had useful data from the current diagnostics, 12 of which also had Xrpin diagnostic data, and 19 shots had MSBeam measurements of diode voltage. Shot series-averaged voltage and current waveforms were then computed, time-aligning the shot data based on the time at which the total cathode current reaches $50 \%$ of its maximum value. The resulting average diode voltage, ion current, and electron current waveforms are shown in Figures 15 (a-c), while the corresponding signals from a QUICKSILVER parasitic load simulation are shown in Figures 15 (d-f). This simulation used an input voltage waveform which included a low voltage precursor to model the vacuum wave precursor observed on SABRE [41]. For this comparison, the QUICKSILVER waveforms have also been time-aligned using the initial ramp-up of the cathode current. Vast differences, appear in the ion current produced in the simulation compared to the experiment, possibly pointing to a lack of understanding of the mechanism by which LiF produces $\mathrm{Li}^{+1}$ ions. Whether $\mathrm{LiF}$ operates by electron-assisted field desorption [23] or by fragmentation of the LiF film leading to local field enhancement [16], in this case a field-threshold 
prescription alone overestimates the amount of lithium current produced. Experimentally, parasitic ion current is observed throughout the pulse, even during the low-voltage precursor, while in the parasitic load simulation, proton emission begins after the lithium current has reached its maximum and sufficient electron heating of the anode face has taken place. The very early parasitic ion production is most likely due to stimulated desorption and partial ionization by surface secondary electrons as has been suggested by Cuneo et al. [3], and is consistent with the dose-independent levels of proton current documented by Sanford et al. [26]. Stimulated desorption is not currently included in the QUICKSILVER parasitic load model but is a straightforward extension, although the uncertainties in fundamental desorption cross-sections would make this extension somewhat ad hoc. Even if stimulated desorption were included, the physics of a neutral or partially-ionized contaminant layer near the anode surface are modeled poorly by the present treatment.

The differences in parasitic load onset point to a fundamental disagreement between the SABRE diode in this configuration and the QUICKSILVER simulations as seen in Figures 15 (c) and (f): an early onset of electron loss during the precursor. As noted in Section IV.C, the diocotron instability broadens the electron sheath but causes very little electron loss to the anode face in this geometry; the transition to the ion mode is required before increased electron loss and rapid heating occur in the simulations. Also, in the simulations electrons populate the sheath primarily by emission from the cathode tip ("Iektip" in Figure 15 (f)) and therefore the rate of electron loss to the anode face relies on the transport of cathode tip electrons across the gap. A different picture of electron current flow is revealed by the experimental measurements. The experimental electron loss, as inferred from X-ray emission from the anode face, is larger than the measured cathode tip electron current. A larger current of electrons flowing at small radii ("Icenter") is measured throughout the pulse. In fact, the measured electron loss current agrees well with the sum of Iektip and Icenter until $\mathrm{t} \cong 55 \mathrm{~ns}$, suggesting that electrons other than cathode tip electrons are responsible for much of the anode heating before and during the initial ion beam emission. 
Estimates of the experimental heating rates and correlations with observed parasitic ion emission are documented by Cuneo et al. [3], showing rapid heating early in the lithium pulse, and similar results are obtained using the measured electron loss plotted here. This suggests that the disagreement between simulation and experiment regarding the lithium production may be due to the electron loss disparity which causes the experimental lithium emission to peak at much lower levels and parasitic ions to be emitted earlier than the simulations predict. In other words, if the processes leading to early electron loss were properly modeled in QUICKSILVER, then better agreement of the parasitic load model results with the experiment would be expected. In the simulations, although space-charge-limited emission of electrons is prescribed for all cathode surfaces (except the artificial extension toward the anode), cathode tip electrons rapidly populate the magnetic flux surface passing through the cathode tip. This electron cloud shields the electric field at the inner cathode coil housing, preventing significant electron emission that would register on Bdot2. The experimental observation of electron current to the anode face from conductor surfaces other than the cathode tip suggests that non-ideal processes (e.g. cathode plasma expansion) are responsible.

\section{PARASITIC LOAD SIMULATIONS OF THE PBFA II RADIAL ION DIODE}

\section{A. ION SOURCE SERIES OF EXPERIMENTS}

The QUICKSILVER simulations described in this section model a series of PBFA II experiments, known as the "ion source series". For the shots considered here, a flat LiF-coated anode was used, with an anode-cathode gap of $1.85 \mathrm{~cm}$, and a $\mathrm{V}_{\text {crit }}$ profile varying from $16.3 \mathrm{MV}$ at the midplane to $19.0 \mathrm{MV}$ at the outer extent of the lithium-emitting region. The LiF source is modeled with a field threshold of $8 \mathrm{MV} / \mathrm{cm}$ for $\mathrm{Li}^{+1}$ emission and a TSCL emission model for protons with $\mathrm{T}_{\mathrm{thr}}=250^{\circ} \mathrm{C}$ and $\tau_{\mathrm{d} 0}=5 \mathrm{~ns}$. Only comparisons with flat anode cases are currently reasonable; the stair-stepped grid treatment of curved surfaces in QUICKSILVER introduces perturbations into the incident electron trajectories to which the electron-surface interaction 


\section{REVISION 2}

model is sensitive.

Figures $16(\mathrm{a}-\mathrm{b})$ show the overall comparison of the QUICKSILVER parasitic load model and PBFA II shot 6148. Large discrepancies appear between the simulated diode currents (total cathode current and ion B-dot monitors) and the experimental waveforms, with the simulation producing 2 to 4 times higher cathode currents (similar to the discrepancies seen in the simulations of SABRE). Most remarkably, the experimental ion B-dot monitor indicates a $\sim 14 \mathrm{~ns}$ delay between the cathode current rise and the ion current rise, compared to the $\sim 5 \mathrm{~ns}$ delay seen in the simulation. According to the simulation model, the delay between these two current measurements is due to the time required to populate the electron sheath (primarily with feed electrons in this geometry) and enhance the electric field at the anode until it exceeds the threshold required for $\mathrm{LiF}$ to emit $\mathrm{Li}^{+1}$ ions. Thus, the longer observed delay could indicate that:

(a) the transport of feed electrons into the diode gap is slower than modeled,

(b) the lithium field threshold is higher than $8 \mathrm{MV} / \mathrm{cm}$, or

(c) the LiF source turn-on is determined by factors other than a simple field threshold.

Resolution of the discrepancies between simulation results and experimental measurements for all applied-B diode configurations is beyond the scope of the parasitic ion emission modeling described here. However, the new QUICKSILVER electron-anode interaction capability can provide some insight as to the possible role of ion source behavior versus feed electron dynamics in the lithium emission discrepancy for this shot series. Regarding hypothesis (a), Figures 17 (a-c) compare measured small aperture $(0.75 \mathrm{~mm}$ diameter) Faraday cup signals to simulation results for PBFA II shot 6148, separating the lithium and proton components. In the simulation, field-threshold lithium emission begins at approximately $40 \mathrm{~ns}$. Meanwhile, portions of the anode face are rapidly heating due to an increasing incident flux of feed electrons. Just prior to $\mathrm{t}=50 \mathrm{~ns}$, parts of the anode face near the midplane begin emitting protons according to the parasitic load model parameters, and register on the "middle" Faraday cup as seen in Figure 17 (b). Due to the electron heating profile (peaked at $Z=1 \mathrm{~cm}$ ), the proton current registered in the 
REVISION 2

"outer" Faraday cup rises somewhat slower as Figure 17 (c) shows. The experimental and simulation results reasonably agree as to the timing of the proton current measured by both Faraday cups, indicating that if the thermal desorption model is correct, then the rate of feed electron loss is being modeled reasonably well. Following this line of reasoning, the timing and location of the onset of proton emission indicates significant electron heating of the anode face earlier than $\mathrm{t}=60 \mathrm{~ns}$, in agreement with the QUTCKSILVER simulation. However, the QUICKSILVER simulation also predicts that, accompanying the electron loss, the sheath electron density and anode electric field have been enhanced such that an $8 \mathrm{MV} / \mathrm{cm}$ threshold source should begin emitting lithium ions. Therefore, feed electron dynamics do not appear to be the determining factor in the experimentally observed lithium emission delay. The Faraday cup measurements also indicate that in this shot series, lithium and proton emission are locally ramping up simultaneously, an effect that cannot be reproduced by the parasitic ion emission model. This behavior may explain the disagreement between the simulation and experiment as to the absolute magnitude of the proton current produced (although Faraday cup aperture closure is another explanation), as well as the low peak lithium current produced in these shots.

To investigate the effect of the field threshold on the lithium emission delay and the overall lithium/proton beam composition (hypothesis (b)) another parasitic load simulation was performed with a lithium emission threshold of $12 \mathrm{MV} / \mathrm{cm}$. Compared to the case with a threshold of $8 \mathrm{MV} / \mathrm{cm}$, this simulation shows a $5.5 \mathrm{~ns}$ delay in the turn-on of the lithium beam relative to the cathode current rise, and a subsequently much lower peak lithium current of $280 \mathrm{kA}$ (compared to $730 \mathrm{kA}$ ) prior to the onset of proton current. Simply raising the lithium emission threshold fails to provide the $\sim 14 \mathrm{~ns}$ ion current delay relative to the cathode current, and although the peak lithium current value is in better agreement with PBFA shot 6148, the simulation does not show simultaneous lithium and proton beam components registering on Faraday cups as was measured (Figures 17 (a-c)). Raising the lithium emission threshold still further (to $16 \mathrm{MV} / \mathrm{cm}$ ) results in the emission of no lithium, because the feed electron heating converts the anode to a 
space-charge-limited proton emitter before the electric field is sufficiently enhanced.

Experimentally-measured electric field profiles for this shot series provide no evidence for a higher field threshold, but do show significant azimuthal asymmetries [42].

Therefore, hypothesis (c) remains a possible explanation for the different experimental behavior observed in the ion source series relative to the simulations and relative to later experimental series. Even with the added level of sophistication provided by the electron deposition-driven parasitic load model, the best 3-D simulations do not reproduce the observed behavior of the ion source for this shot series. As with the SABRE comparisons, other missing physical effects such as anode and cathode plasma expansion are likely contributing to the overall discrepancy in voltage and current histories.

\section{B. PARASITIC LOAD SERIES OF EXPERIMENTS}

The QUICKSILVER simulations described in this section are a direct follow-on to those previously published [13], adding the new electron-anode interaction capabilities. This configuration corresponds to the "parasitic load series"[17] of PBFA II shots, which used flat (15 $\mathrm{cm}$ constant radius) $\mathrm{LiF}$ anodes, a $1.8 \mathrm{~cm}$ anode-cathode gap, fairly uniform magnetic insulation at a $V_{\text {crit }}$ of $17.6 \mathrm{MV}$ (a profile quite different from the earlier ion source series), various current diagnostics, and detailed spectroscopic measurements of the electric field profile in the gap. The earlier QUICKSILVER results showed good agreement of the accelerator model with the observed diode current rise and reasonable agreement of the field-threshold model with the measured lithium current rise. For the simulation described here, the threshold field for $\mathrm{Li}^{+1}$ emission is 8 $\mathrm{MV} / \mathrm{cm}$ and the TSCL emission model for protons uses $\mathrm{T}_{\mathrm{thr}}=250^{\circ} \mathrm{C}$ and $\tau_{\mathrm{d} 0}=5 \mathrm{~ns}$. This simulation modeled 1/16th of the azimuthal extent and half of the height of the diode, with symmetry boundary conditions applied at $\mathrm{z}=0$. The accelerator model driving the diode has been documented previously [13]. 
A key feature of the PBFA II diode which appears in the simulations is the contribution of feed electrons in populating the sheath and assisting the onset of field-threshold lithium emission. These feed electrons are less well magnetically-insulated than cathode tip electrons and are readily lost to the anode face under the influence of diocotron instabilities, before any ion emission has occurred. Figure 18 shows the total cathode current, the primary feed electron loss current to the anode face, and the maximum anode temperature time histories. As in the SABRE simulations, the reflected electron loss current (not shown) is nearly identical to the primary electron current, and increases the anode surface heating rate. The most rapid heating occurs between $\mathrm{t}=40 \mathrm{~ns}$ and $\mathrm{t}=52 \mathrm{~ns}$, with the maximum anode temperature rising by $350^{\circ} \mathrm{C}$. A temperature snapshot at $\mathrm{t}=52 \mathrm{~ns}$ appears in Figure 19, showing the band of high electron flux and concentrated heating at $\mathrm{z} \cong 1 \mathrm{~cm}$, resulting in temperatures exceeding the $250^{\circ} \mathrm{C}$ threshold for 0.5 $\leq \mathrm{z} \leq 2.0 \mathrm{~cm}$.

The predicted location of the initial proton emission and lithium shut-off is close to the midplane of the diode, and therefore should be readily measurable by Faraday cups filtered to distinguish lithium from protons. PBFA II shot 6411 utilized an array of azimuthally-spaced, large aperture ( $5 \mathrm{~mm}$ diameter) Faraday cups centered on the midplane, two of which had $100 \mu \mathrm{m}$ Mylar filters (assumed to pass protons with energies above $2.7 \mathrm{MeV}$ ), and two of which had 12 $\mu \mathrm{m}$ Mylar filters (assumed to pass essentially all protons and those lithium ions with energies above $4.5 \mathrm{MeV}$ ). The $12 \mu \mathrm{m}$ filtered cup also responds to carbon accelerated as $\mathrm{C}^{2+}$, but this contribution is expected to be $<6 \%$ and peaks later in the pulse [17], and so is ignored for this comparison. The lithium current is derived by subtracting the proton-only cup signal from the 12 $\mu \mathrm{m}$ filtered cup signal, then dividing by a stripping factor of 3.0 applicable to lithium above $\sim 7.5$ $\mathrm{MeV}$. Figures 20 (a-c) compare the total ion current (from B-dot measurements), lithium current, and proton current for this simulation and shot 6411 . Figures $20(a-b)$ show that the initial (lithium) ion current rise is remarkably well reproduced in the simulation from $\mathrm{t}=40 \mathrm{~ns}$ to $\mathrm{t}=50$ ns. The parasitic load model in this case also agrees quantitatively with the peak lithium current 
prior to shut-off $(\sim 1 \mathrm{MA})$, the peak level of proton current produced ( $2 \mathrm{MA})$, and with the approximate shape of the lithium pulse. The cell-by-cell instantaneous switch from FT lithium emission to SCL proton emission causes a more sudden transition than is observed, and this leads to the overproduction of total ion current seen in Figure 20 (a), and the more rapid rise in the proton current seen in Figure 20 (c). The key simulation results concerning particle currents are that the calculated electron flux leads to local anode heating above the known plasma formation threshold, causing the lithium current to decay, replaced by a proton current, and that the currents of the two ion species are in good quantitative agreement with measurements. Although the electron loss to the anode face is not experimentally measured, the fact that the simulation correctly predicts the onset of proton current indirectly implies that the local heating rates, and therefore the electron flux profile, are modeled reasonably well if the thermal desorption model is correct.

Figures 21 (a-c) compare the simulated diode voltage and current, diode impedance, and lithium impedance for the present parasitic load simulation, the baseline simulation previously documented [13], and measurements from shot 6411 . The diode voltage is monitored by a Rutherford scattering magnetic spectrometer, and so measurements actually begin at $\mathrm{t} \cong 45 \mathrm{~ns}$ as significant lithium ion emission occurs. The experimental diode voltage prior to this is calculated using a flow impedance related to the accelerator feed impedance and anode and cathode currents near the diode[17]. The rapid drop in the simulated diode voltage at $\mathrm{t} \cong 52 \mathrm{~ns}$ in the parasitic load simulation is due to the onset of the proton current which rises to $\sim 2 \mathrm{MA}$ in about $10 \mathrm{~ns}$. Later in time, the parasitic load simulation voltage roughly agrees with the baseline simulation, and both simulated voltages remain significantly above the measured voltage. Experimentally, the diode voltage continues to dectine from $50 \mathrm{~ns}$ forward while the diode current continues to increase, an example of the collapsing impedance typical of PBFA II lithium shots. Because the simulations cannot model the expansion of dense anode and cathode plasmas in the feed or diode which contribute to impedance collapse, the diode impedance in the parasitic load simulation levels off 
at about 4 ohms from $60 \mathrm{~ns}$ on, although the very early stages of impedance collapse are reproduced better by the parasitic load model than by the baseline simulation. Certainly, the lithium impedance is better modeled by the parasitic load simulation as Figure 21 (c) shows, which is to be expected given the level of agreement with the rate of lithium current decline. If the experimental voltage decline is due to anode plasma expansion through the compressed magnetic field [8], then an initial expansion velocity of $\sim 0.2 \mathrm{~mm} / \mathrm{ns}$ is required, for the (simulation) $6.5 \mathrm{~T}$ anode magnetic field. This velocity implies that the assumption of a thin anode plasma layer comparable to the near-anode $\Delta r$ grid dimension $(0.4 \mathrm{~mm})$ is violated within a few nanoseconds anyway. Overall, the fidelity of the simulation voltage and current histories has been extended by at least $5 \mathrm{~ns}$ with the addition of the relatively simplistic parasitic load model, with much greater improvement if the particle-specific currents are considered. In other words, the parasitic load model contains the correct physics to model the production of proton current due to electron bombardment, but does not explain the observed impedance collapse which requires a treatment of the expansion of dense electrode plasmas.

Time- and space-resolved measurements of the electric field in the anode-cathode gap were made for PBFA II shot 6411, using the Stark shift of Li I 2s-2p emission [22]. Since the diagnostic relies on light emission from neutral lithium atoms, useful measurements can only be made on lines of sight passing through regions into which neutral lithium atoms have been transported or in which neutral lithium has been created by charge exchange (e.g. with neutral contaminants [43]). Figures 22 (a-f) compare radial profiles of the electric field magnitude for the parasitic load simulation, the baseline simulation, and the measured data points from the Stark shift diagnostic. The simulated profiles were averaged over the half-height of the lithium emission region $(5 \mathrm{~cm})$, and all three profiles used a $2 \mathrm{~ns}$ time window average centered about the time indicated on each plot. For the measured data, the horizontal line indicates the line-of-sight diameter $(2 \mathrm{~mm})$, while the vertical error bars are one standard deviation. During the lithium current ramp-up phase ( $47 \mathrm{~ns}$ and $51 \mathrm{~ns}$ snapshots), the parasitic load simulation and baseline 
simulation profiles are similar and match the near-anode field measurements reasonably well. Differences in the two simulation profiles appear after $\mathrm{t} \sim 55 \mathrm{~ns}$, the time at which the lithium current peaks and the first protons appear in the parasitic load simulation. Because the total ion current for the two simulations is similar, the parasitic load simulation E-field profiles (and sheath electron density profiles) are lower near the anode than in the baseline simulation due to the influence of the higher-velocity proton component. In general, the line-of-sight average electric field is lower and less strongly peaked in the parasitic load simulation which brings it into better agreement with the measured profiles than the baseline simulation. However, after $\mathrm{t} \sim 55 \mathrm{~ns}$, the measured data reflect the decaying diode voltage which is not reproduced in the parasitic load simulation, as was seen in Figure 21(a).

Although the new models have improved the level of agreement between the simulations and the parasitic load series of experiments, they reveal no clear explanation for the much different behavior seen in the earlier ion source series. Since several months passed and machine hardware changes occurred between the two shot series, there are variables (e.g. 3-D hardware asymmetries, anode sleeve preparation) which are unaccounted for in the simulations presented here. It is interesting that the behavior of the more recent shot series (the parasitic load series) is better reproduced by the simulations, given that much experimental effort in the intervening months sought to reduce systematic effects such as anode substrate azimuthal asymmetry.

\section{CONCLUSIONS}

To increase the realism of, and information derived from, three-dimensional PIC simulations of applied-B ion diodes, the physics of electron-anode interactions have been included. Tabulated resúlts from Monte Carlo electron-photon transport calculations are now available to assess, within the PIC simulation, the effects of electron reflection and electron energy deposition in anode materials. Because these effects occur only for killed particles, the computational overhead is small relative to the time spent in tracking the history of the particles. 


\section{REVISION 2}

Simulations of the SABRE extraction ion diode, using the new electron-anode interaction capabilities, led to the following primary results:

(a) Despite the relatively high overall reflection coefficient (70\%), the lifetime of reflected electrons is short enough that they contribute little to the space charge in the diode gap, and thus have a negligible direct effect on the gross diode behavior.

(b) In extraction geometry, the transition from the diocotron mode to the ion mode as the dominant sheath instability is accompanied by a sudden increase in the electron loss current to the anode face. The electron flux spatial profile is related to the ion mode structure, leading to peak-to-average temperature ratios of approximately 5 .

(c) The rapid anode heating occurring after the ion mode transition leads to complete desorption of the $15-20 \mathrm{kcal} / \mathrm{mole}$ contaminant inventory within $10 \mathrm{~ns}$ for the regions of peak electron flux, and $10 \%$ desorption in other regions. Reflected electrons significantly affect the spatial temperature distribution, due to their transverse motion between impacts.

(d) A parasitic ion emission model, based upon the calculated anode heating and empirical plasma formation temperature thresholds, demonstrates the cessation of lithium current, the onset of parasitic ion current, and the late-time decrease in electron loss typical of SABRE shots. However, large discrepancies in the amount of lithium current produced and the sources of diode electrons indicate that other non-ideal effects are dominating the behavior of the real diode. In particular, the presence of earlier electron loss on SABRE suggests that a layer of neutral or partially-ionized contaminants may form near the anode, a situation modeled poorly by the present parasitic ion emission model. This conclusion is consistent with inferred lithium charge-exchange probabilities of near unity early in SABRE shots.

Simulations of the ion source series on the PBFA II radial diode show:

(a) The experiments exhibit a significant delay in the lithium current onset which cannot be reproduced by simple changes in simulation parameters (e.g. electron and lithium emission field thresholds).

(b) The timing of the proton current onset is well reproduced, supporting the simulated electron loss and anode heating results.

(c) For the diode configuration of this shot series, other non-ideal effects, perhaps engendered 
by hardware asymmetries or ion source preparation, were possibly dominating the observed behavior.

Building on earlier simulations of the parasitic load series of experiments on PBFA II, an assessment of the electron energy deposition shows that:

(a) Unlike the extraction geometry, in the radial geometry, electrons born in the feed account for the bulk of the anode heating, particularly early in the power pulse. As simulations with the temperature-threshold parasitic ion emission model demonstrate, this feed electron deposition causes rapid anode heating during the lithium beam ramp-up phase, well before the predicted transition to the ion mode.

(b) The simulated timing of the lithium current onset and decline, and the proton current onset, as well as the absolute amounts of the two beam components, agree well with the experimental Faraday cup measurements.

(c) The results of the parasitic ion emission model yield electric field profiles which evolve in better agreement with spectroscopic measurements than the baseline simulations [22].

(d) The late time fidelity of the simulations is limited by the lack of an adequate model of the physics of diode impedance collapse due to anode and cathode plasma expansion. The fact that better overall agreement of the simulations is seen with the more recent parasitic load series is possibly due to improvements in the diode configuration (making the real diode more "ideal"), an ongoing process during the PBFA II experiments.

In general, the addition of electron-anode interaction physics has improved the value and fidelity of diode simulations. However, the results presented in this paper have also demonstrated the limitations of these simulations in predicting or explaining the behavior of applied-B ion diode experiments. Much of the discrepancy is likely due to uncertainties in the modeling of ion emission processes; the modeling of high energy electron-material interactions is based on wellestablished physics. Therefore, the detailed modeling of electron-anode interactions (reflection, energy deposition, and photon production) will be a valuable addition to other applications of three-dimensional PIC simulations, such as magnetically-insulated transmission lines, post-hole convolute power transmission components for high-power fast Z-pinch devices, electron diodes 
REVISION 2

for radiography, and high-power microwave devices.

\section{ACKNOWLEDGMENTS}

We wish to thank the QUICKSILVER team (D. B. Seidel, M. L. Keifer, R. S. Coats, L. P. Mix, and M. F. Pasik) for ongoing development of the QUICKSILVER and PFIDL simulation/ analysis tools, J. A. Halbleib and R. Kaye for assistance with the ITS codes, and D. L. Cook and J. P. Quintenz for programmatic support. Sandia is a multiprogram laboratory operated by Sandia Corporation, a Lockheed Martin Company, for the United States Department of Energy under Contract DE-AC04-94AL85000. 
REVISION 2

\section{REFERENCES}

1. T. A. Mehlhorn, IEEE Trans. Plasma Sci. 25, 1336 (1997).

2. See National Technical Information Service Document No. PB95-144317 (T. A. Mehlhorn, J. E. Bailey, G. A. Chandler, R. S. Coats, M. E. Cuneo, M. S. Derzon, M. P. Desjarlais, R. J. Dukart, A. B. Filuk, T. A. Haill, H. C. Ives, D. J. Johnson, R. J. Leeper, T. R. Lockner, C. W. Mendel, P. R. Menge, L. P. Mix, A. R. Moats, W. B. Moore, T. D. Pointon, J. W. Poukey, J. P. Quintenz, S. E. Rosenthal, D. C. Rovang, C. L. Ruiz, S. A. Slutz, W. A. Stygar, and D. F. Wenger, in Proceedings of the 10th International Conference on High Power Particle Beams, San Diego, CA, 1994, p. 53). Copies may be ordered from NTIS, Springfield, Virginia 22161.

3. M. E. Cuneo, P. R. Menge, D. L. Hanson, W. E. Fowler, M. A. Bernard, G. R. Ziska, A. B. Filuk, T. D. Pointon, R. A. Vesey, D. R. Welch, J. E. Bailey, M. P. Desjarlais, T. R. Lockner, T. A. Mehlhorn, S. A. Slutz, and M. A. Stark, IEEE Trans. Plasma Sci. 25, 229 (1997).

4. M. E. Cuneo, P. R. Menge, D. L. Hanson, W. E. Fowler, M. A. Bernard, G. R. Ziska, A. B. Filuk, J. E. Bailey, M. P. Desjarlais, T. R. Lockner, T. J. Nash, D. D. Noack, and S. A. Slutz, in Proceedings of the 10th IEEE Pulsed Power Conference, Albuquerque, NM, July 1995, p. 640 .

5. D. R. Welch, M. E. Cuneo, C. L. Olson, and T. A. Mehlhorn, Phys. Plasmas 3, 2113 (1996).

6. S. A. Slutz and D. B. Seidel, J. Appl. Phys. 59, 2685 (1986).

7. M. P. Desjarlais, Phys. Rev. Lett. 59, 2295 (1987).

8. M. P. Desjarlais, Phys. Fluids B 1, 1709 (1989).

9. D. B. Seidel, M. L. Kiefer, R. S. Coats, T. D. Pointon, J. P. Quintenz, and W. A. Johnson, in Proceedings of the Europhysics Conference on Computational Physics, Amsterdam, 1990, edited by A. Tenner (World Scientific, Singapore, 1991), p. 475.

10. M. P. Desjarlais, T. D. Pointon, D. B. Seidel, R. S. Coats, M. L. Kiefer, J. P. Quintenz, and S. A. Slutz, Phys. Rev. Lett. 67, 3094 (1991).

11. T. D. Pointon, M. P. Desjarlais, D. B. Seidel, S. A. Slutz, R. S. Coats, M. L. Kiefer, and J. P. Quintenz, Phys. Plasmas 1, 429 (1994). 
REVISION 2

12. R. H. Levy, Phys. Fluids 8, 1288 (1965).

13. T. D. Pointon and M. P. Desjarlais, J. Appl. Phys. 80, 2079 (1996).

14. See National Technical Information Service Document No. PB92-206168 (M. P. Desjarlais and T. D. Pointon, in Proceedings of the 9th International Conference on High Power Particle Beams, Washington, DC, May 1992, edited by D. Mosher and G. Cooperstein, p. 775). Copies may be ordered from NTIS, Springfield, Virginia 22161.

15. R. W. Lemke and S. A. Slutz, Phys. Plasmas 2, 549 (1995).

16. S. A. Slutz, R. W. Lemke, T. D. Pointon, M. P. Desjarlais, D. J. Johnson, T. A. Mehlhorn, A. Filuk, and J. Bailey, Phys. Plasmas 3, 2175 (1996).

17. D. J. Johnson, S. E. Rosenthal, R. S. Coats, M. P. Desjarlais, T. R. Lockner, T. A. Mehlhorn, T. D. Pointon, C. L. Ruiz, W. A. Stygar, S. A. Slutz, and D. F. Wenger, Laser and Particle Beams 16, 185 (1998).

18. H. Bluhm, P. Hoppe, M. Althaus, H. Bachmann, W. Bauer, K. Baumung, L. Buth, H. U. Karow, H. Laqua, D. Rusch, E. Stein, and O. Stoltz, in Ref. 14, p. 51; J. B. Greenly, R. K. Appartaim, J. C. Olson, and L. Brissette, in Ref. 2, p. 398.

19. N. R. Pereira, J. Appl. Phys. 54, 6307 (1983).

20. D. Mosher, G. Cooperstein, D. V. Rose, and S. B. Swanekamp, Bull. Am. Phys. Soc. 40, 1751 (1995).

21. J. P. Quintenz and M. M. Widner, J. Appl. Phys. 51, 4688 (1980).

22. J. E. Bailey, A. B. Filuk, A. L. Carlson, D. J. Johnson, P. Lake, E. J. McGuire, T. A. Mehlhorn, T. D. Pointon, T. J. Renk, W. A. Stygar, and Y. Maron, Phys. Rev. Lett. 74, 1771 (1995).

23. See National Technical Information Service Document No. SAND95-1794 (T. A. Green, R. W. Stinnett, R. A. Gerber, P. F. McKay, M. F. Lopez, R. A. Anderson, T. D. Pointon, L. J. Lorence, Jr., J. A. Halbleib, A. B. Filuk, J. A. Panitz, and P. R. Schwoebel, "Production of Lithium Positive Ions from LiF Thin Films on the Anode in PBFA II," Sandia National Laboratories Report No. SAND95-1794, September, 1995). Copies may be ordered from 
NTIS, Springfield, Virginia 22161.

24. J. E. Bailey, A. B. Filuk, A. L. Carlson, D. J. Johnson, P. Lake, E. J. McGuire, T. A. Mehlhorn, T. D. Pointon, T. J. Renk, W. A. Stygar, Y. Maron, and E. Stambulchik, Laser and Particle Beams 14, 543 (1996).

25. D. W. Swain, S. A. Goldstein, J. G. Kelly, and G. R. Hadley, J. Appl. Phys. 46, 4604 (1975); J. G. Kelly, S. A. Goldstein, and D. W. Swain, J. Appl. Phys. 46, 4726 (1975);

A. E. Blaugrund, G. Cooperstein, and S. A. Goldstein, Phys. Fluids 20, 1185 (1977).

26. T. W. L. Sanford, J. A. Halbleib, J. W. Poukey, A. L. Pregenzer, R. C. Pate, C. E. Heath, R. Mock, G. A. Mastin, D. C. Ghiglia, T. J. Roemer, P. W. Spence, and G. A. Proulx, J. Appl. Phys. 66, 10 (1989).

27. See, for example, R. I. Masel, Principles of Adsorption and Reaction on Solid Surfaces (Wiley, New York, 1996), p. 507 and references therein.

28. See National Technical Information Service Document No. SAND91-1634 (J. A. Halbleib, R. P. Kensek, T. A. Mehlhorn, G. D. Valdez, S. M. Seltzer, and M. J. Berger, "ITS Version 3.0: The Integrated TIGER Series of Coupled Electron/Photon Monte Carlo Transport Codes," Sandia National Laboratories Report No. SAND91-1634, March, 1992). Copies may be ordered from NTIS, Springfield, Virginia 22161.

29. D. Heifetz, D. Post, M. Petravic, J. Weisheit, and G. Bateman, "A Monte-Carlo Model of Neutral-Particle Transport in Diverted Plasmas," J. Comp. Phys. 46, 309-327 (1982); M. J. Berger, in Methods of Computational Physics, Vol. 1 (Academic, New York, 1963), p. 135. 30. P. R. Menge and M. E. Cuneo, IEEE Trans. Plasma Sci. 25, 252 (1997).

31. D. J. Johnson, E. J. T. Burns, J. P. Quintenz, K. W. Bieg, A. V. Farnsworth, Jr., L. P. Mix, and M. A. Palmer, J. Appl. Phys. 52, 168 (1981); R. Pal and D. Hammer, Phys. Rev. Lett. 50, 732 (1983); E. W. Gray, J. Appl. Phys. 58, 132 (1985); Y. Maron, E. Sarid, O. Zahavi, L. Perelmutter, and M. Sarfaty, Phys. Rev. A 39, 5842 (1989); Y. Maron, M. Sarfaty, L. Perelmutter, O. Zahavi, M. E. Foord, and E. Sarid, Phys. Rev. A 40, 3240 (1989).

32. The Cray J90 system is described at http://www.cray.com/products/systems/crayj90/ 


\section{REVISION 2}

overview.html.

33. D. R. Welch, C. L. Olson, and T. W. L. Sanford, Phys. Plasmas 1, 764 (1994).

34. DYNAID is a 1-D hybrid code using PIC methods in low collisionality regions and the multifluid model of weakly-ionized plasma described in P. W. Rambo and J. Denavit, J. Comp. Phys. 92, 185 (1991); J. Comp. Phys. 98, 317 (1992).

35. J. P. Quintenz, D. B. Seidel, M. L. Kiefer, T. D. Pointon, R. S. Coats, S. E. Rosenthal, T. A. Mehlhorn, M. P. Desjarlais, and N. A. Krall, Laser and Particle Beams 12, 283 (1994).

36. G. M. McCracken, R. S. Barton, and W. Dillon, Suppl. Nuovo Cimento 5, 146 (1967).

37. M.-H. Achard, R. Calder, and A. Mathewson, Vacuum 29, 53 (1979).

38. R. A. Anderson and J. P. Brainard, J. Appl. Phys. 51, 1414 (1980).

39. R. A. Langley, J. Nucl. Mater. 128-129, 622 (1984).

40. S. A. Slutz, T. A. Mehlhorn, J. E. Maenchen, C. Ruiz, and J. R. Woodworth, J. Appl. Phys. 62, $16(1987)$.

41. M. E. Cuneo, D. L. Hanson, J. W. Poukey, P. R. Menge, M. E. Savage, J. R. Smith, and M. A. Bernard, in Ref. 2, p. 399.

42. J. E. Bailey, A. L. Carlson, A. B. Filuk, D. J. Johnson, P. Lake, E. J. McGuire, T. A. Mehlhorn, T. D. Pointon, T. J. Renk, and Y. Maron, in Ref. 2, p. 57.

43. A. B. Filuk, J. E. Bailey, A. L. Carlson, D. J. Johnson, P. Lake, T. A. Mehlhorn, L. P. Mix, T. J. Renk, W. A. Stygar, and Y. Maron, Phys. Rev. Lett. 77, 3557 (1996). 
REVISION 2

\section{FIGURE CAPTIONS}

FIGURE 1. Simplified drawing of the (a) SABRE extraction ion diode, and (b) PBFA II radial ion diode configuration, indicating the locations of selected diagnostics. In (a), the F-cups and Rutherford scattering foil are separated azimuthally.

FIGURE 2. Contour plot of total electron reflection coefficient, $R(E, \theta)$, as a function of the incident electron energy and polar angle, as calculated by ITS.

FIGURE 3. ITS calculated reflected electron distribution $P\left(E^{\prime}, \theta^{\prime}\right) \mathrm{dE} d \Omega$ for a $4 \mathrm{MeV}$ primary electron incident at $80^{\circ}$ to normal.

FIGURE 4. ITS calculated electron energy deposition (MeV/cm/electron) in a LiF/SS304 anode as a function of depth, for a $4 \mathrm{MeV}$ primary electron incident at $80^{\circ}$.

FIGURE 5. Azimuthal (r-z) slice of the conductor geometry and applied-B streamlines used in QUICKSILVER simulations of the SABRE diode. The 3-D model actually extends to $\mathrm{Z}=-30 \mathrm{~cm}$, where a 1-D transmission line model is connected.

FIGURE 6. Azimuthally-averaged profiles of (feed+diode) primary and reflected electrons in a simulation of SABRE. Note that the y-axis is logarithmic, showing the dominance of primary over reflected electrons in contributing to the space charge.

FIGURE 7. Normalized electron fluxes to the anode surface as a function of radius for a SABRE simulation. For this applied-B geometry, the primary flux peaks at the inner anode radius; the reflected electron flux is more uniformly distributed.

FIGURE 8. Time history of the primary (solid) and reflected (dotted) electron currents to the anode face for a SABRE simulation. 
FIGURE 9. Time histories of (a) the $m=8$ mode from a spatial FFT of the azimuthal electric field, (b) the azimuthal microdivergence of the lithium beam, (c) electron loss power striking the anode face, and (d) surface temperatures in the stainless steel anode substrate. Plot (d) shows the overall maximum temperature, maximum temperature in the lithium-emitting portion, and an "average" temperature which would result from a uniform spatial distribution of the power plotted in (c).

FIGURE 10. Snapshots of $(\mathrm{a}, \mathrm{c}, \mathrm{e})$ the volumetric electron heating rate $\left(\mathrm{GW} / \mathrm{cm}^{3}\right)$ in the top $10 \mu \mathrm{m}$ of stainless steel, and $(b, d, f)$ the resulting temperature profile, for a SABRE simulation. Each plot represents a head-on view of the annular anode face; 1/4 of the azimuthal extent was modeled.

FIGURE 11. Time history of stimulated (dotted) and thermal (solid) desorption frequencies (azimuthally-averaged at $\mathrm{r}=4.3 \mathrm{~cm}$ ), calculated using QUICKSILVER/TTS results for electron flux and stainless steel temperature.

FIGURE 12. Time history of the desorbed contaminant fraction for three desorption models: (a) constant binding energy $Q=15 \mathrm{kcal} / \mathrm{mole}$, (b) binding energy increasing from 15 to $20 \mathrm{kcal} / \mathrm{mole}$ as desorption proceeds, and (c) second-order desorption using parameters representing the $\mathrm{H}_{2}$ surface recombination process. An overall maximum and a maximum in the lithium-emitting portion of the anode face are plotted in each case.

FIGURE 13. Time histories of (a) diode voltage, (b) ion currents, and (c) electron loss to the anode face, for a simulation of SABRE using the QUICKSILVER parasitic load model, compared to the baseline simulation results.

FIGURE 14. Snapshots from the parasitic load simulation of $(\mathrm{a}, \mathrm{c}, \mathrm{e})$ electron flux striking the anode face, and $(\mathrm{b}, \mathrm{d}, \mathrm{f}) \mathrm{Li}^{+1}$ (open) and proton (filled) particles within $1 \mathrm{~mm}$ of the 
anode face. These snapshots show the feedback of the switch to space-chargelimited proton emission on the local electron loss.

FIGURE 15. Comparison of time histories of $(\mathrm{a}, \mathrm{d})$ diode voltage, $(\mathrm{b}, \mathrm{e})$ cathode and ion currents, and $(\mathrm{c}, \mathrm{f})$ total cathode, electron loss, cathode tip, and center currents. Plots (a-c) represent the average over a series of SABRE experiments, while plots (d-f) are the results of a QUICKSILVER simulation which included a precursor in the input voltage waveform. For comparison, the QUICKSILVER results are time-shifted to align the initial cathode current rise. (Note the difference in scales of adjacent plots.)

FIGURE 16. For PBFA II ion source series configuration, the (a) diode voltage and total cathode current, and (b) the total ion current as measured by ion B-dot monitors, for a QUICKSILVER parasitic load simulation (solid) and shot 6148 experimental results (dotted).

FIGURE 17. Small Faraday cup measurements, as calculated by QUICKSILVER and measured for shot 6148 , for (a) lithium component, middle and outer F-cups, (b) proton component, middle F-cups, and (c) proton component, outer F-cups.

FIGURE 18. Total cathode current, maximum anode temperature, and the primary feed electron current to the anode face for a QUICKSILVER simulation of the PBFA II radial diode, parasitic load series configuration.

FIGURE 19. Snapshot of the $(\phi-z)$ anode temperature field at $t=52 \mathrm{~ns}$. In this geometry, $r$ is constant on the anode face, one-half of the vertical extent of the diode is modeled (the midplane is at $\mathrm{z}=0$ ), and $1 / 16$ of the azimuthal extent is modeled.

FIGURE 20. Time histories of (a) total ion current as measured by ion B-dot monitors, (b) total lithium current, and (c) total proton current, for the QUICKSILVER parasitic load 
simulation (solid), compared to experimental measurements (dotted) for PBFA II shot 6411 .

FIGURE 21. Time histories of (a) diode voltage and total cathode current, (b) diode impedance, and (c) lithium impedance, for QUICKSIL VER parasitic load simulation (solid), baseline simulation (dot-dash), and PBFA II shot 6411 (dotted) results.

FIGURE 22. Profiles of the electric field magnitude for QUICKSILVER parasitic load simulation (solid), baseline simulation (dotted), and PBFA II shot 6411 spectroscopic results (connected data points with error bars). The $47 \mathrm{~ns}$ snapshot is early in the lithium current rise, by $67 \mathrm{~ns}$ the lithium current has greatly subsided and the parasitic proton current is near its peak value. 

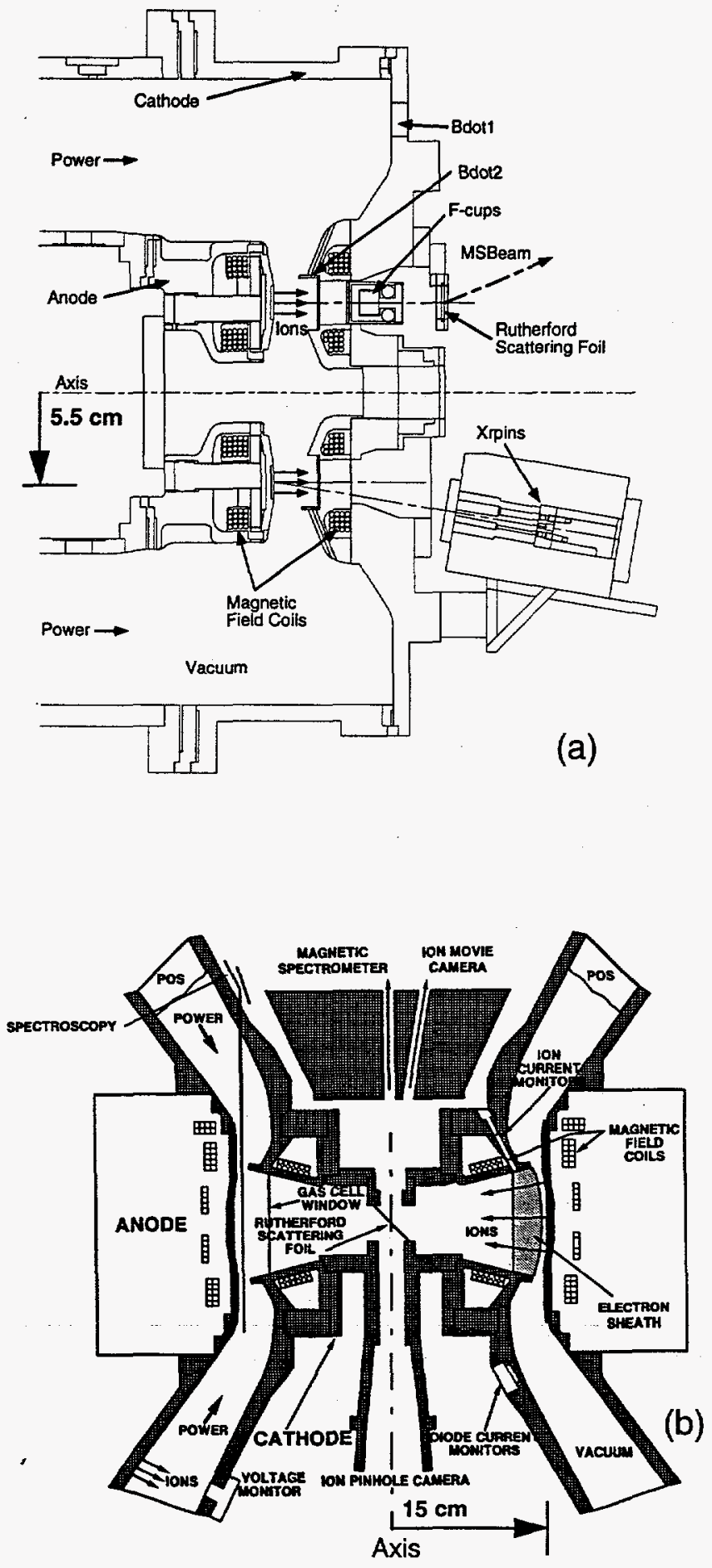

R. Vesey

Phys. Plasmas
Figure 1. 


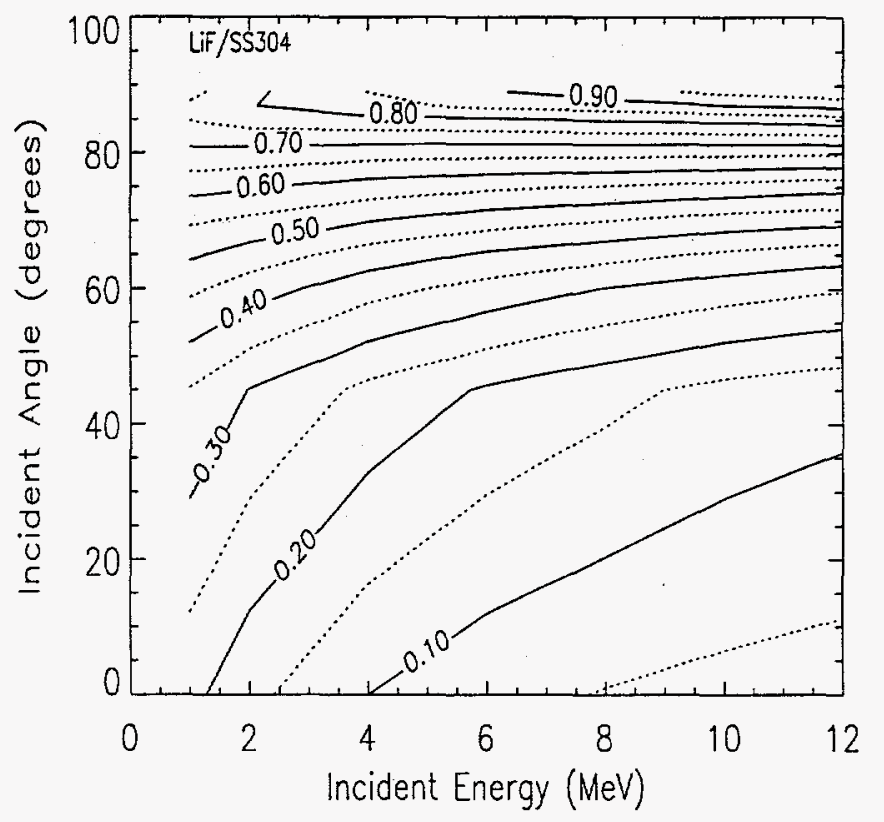

R. Vesey

Phys, Plasmas

Figure 2. 


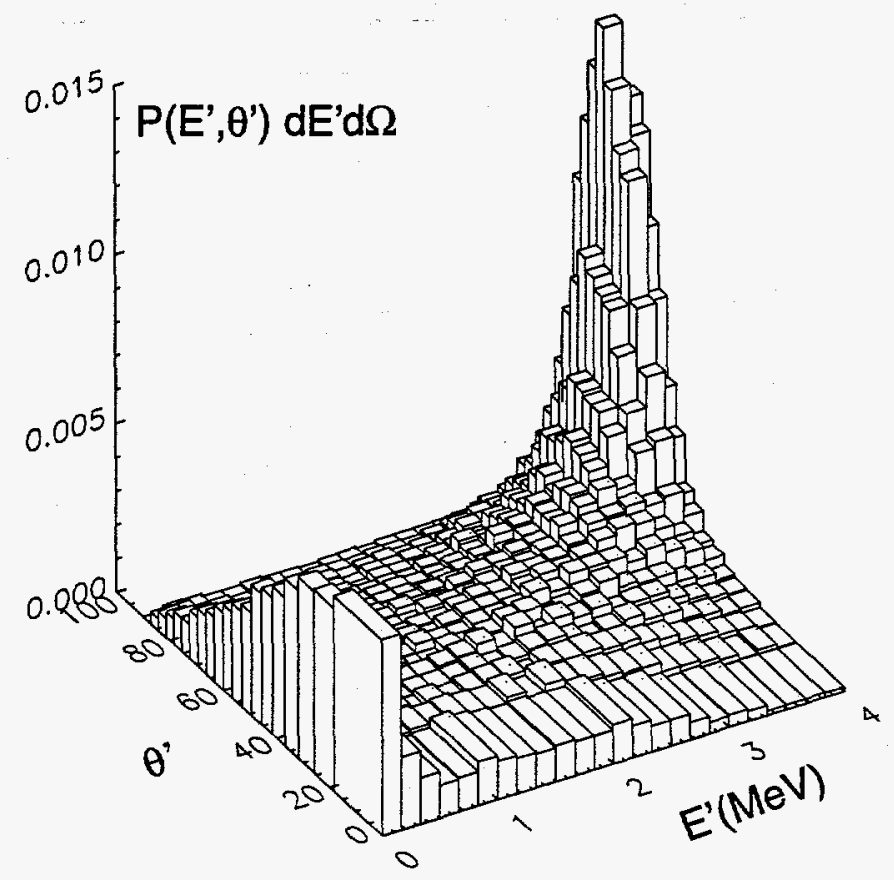

Figure 3. 


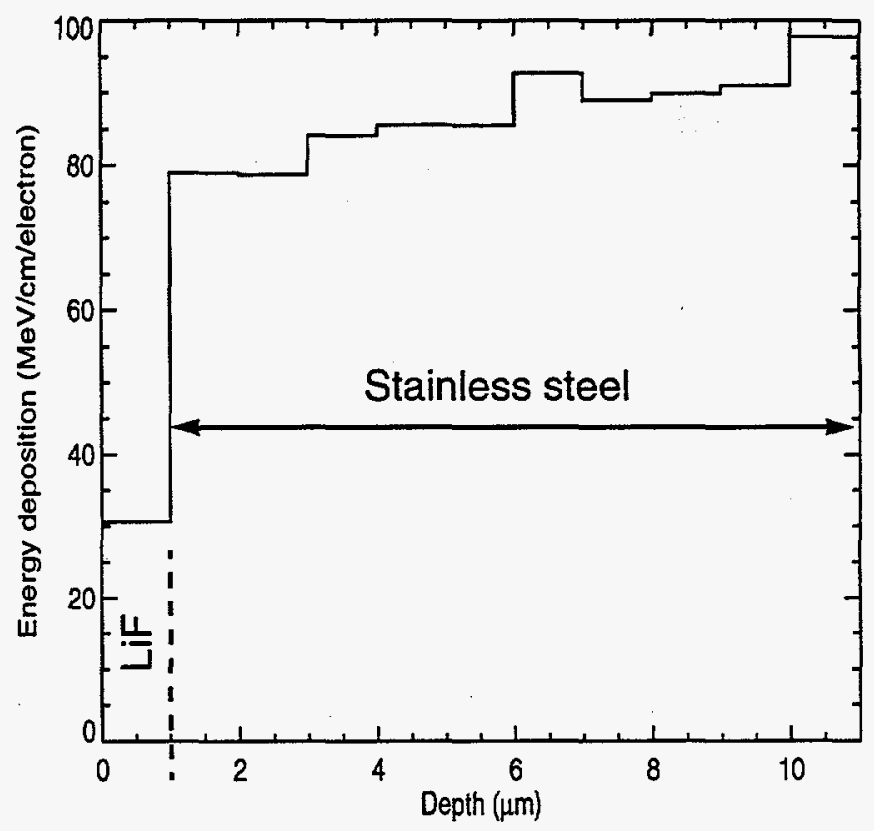

R. Vesey

Phys. Plasmas

Figure 4. 


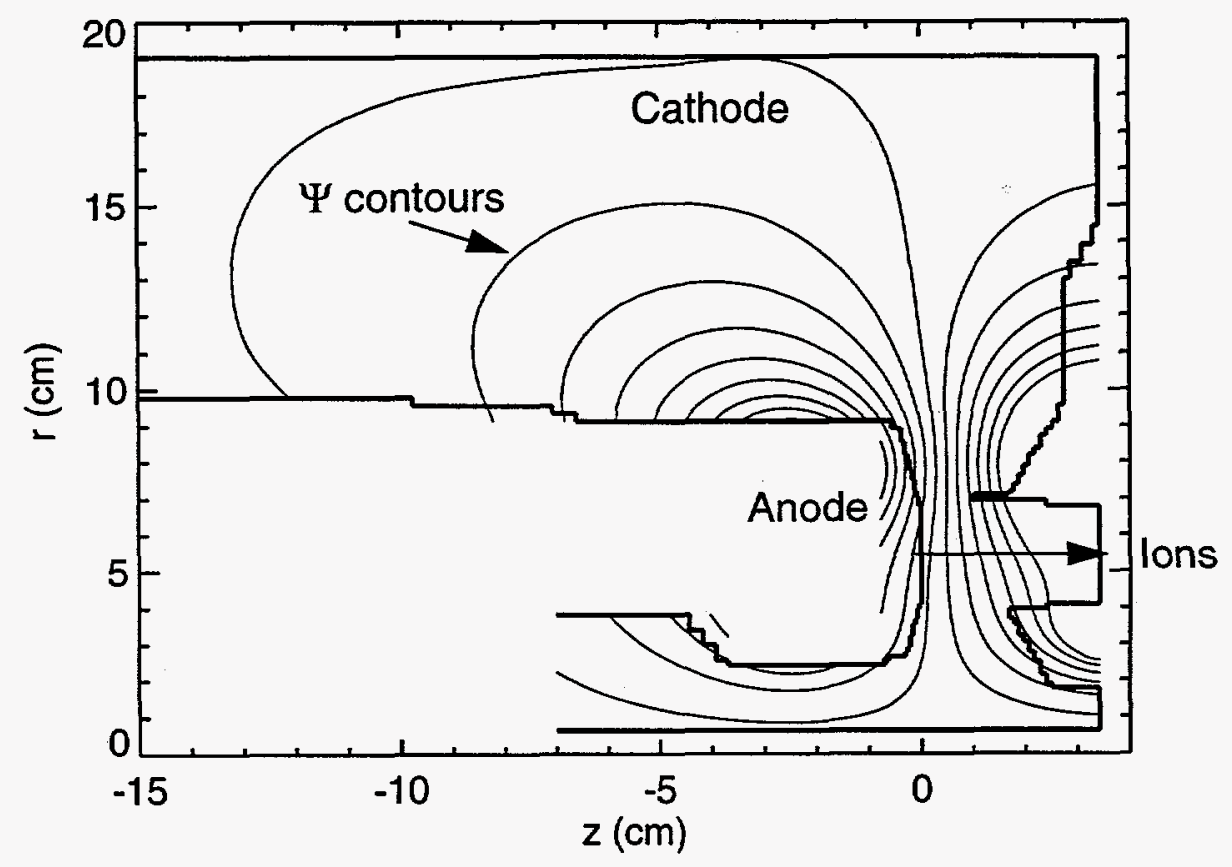




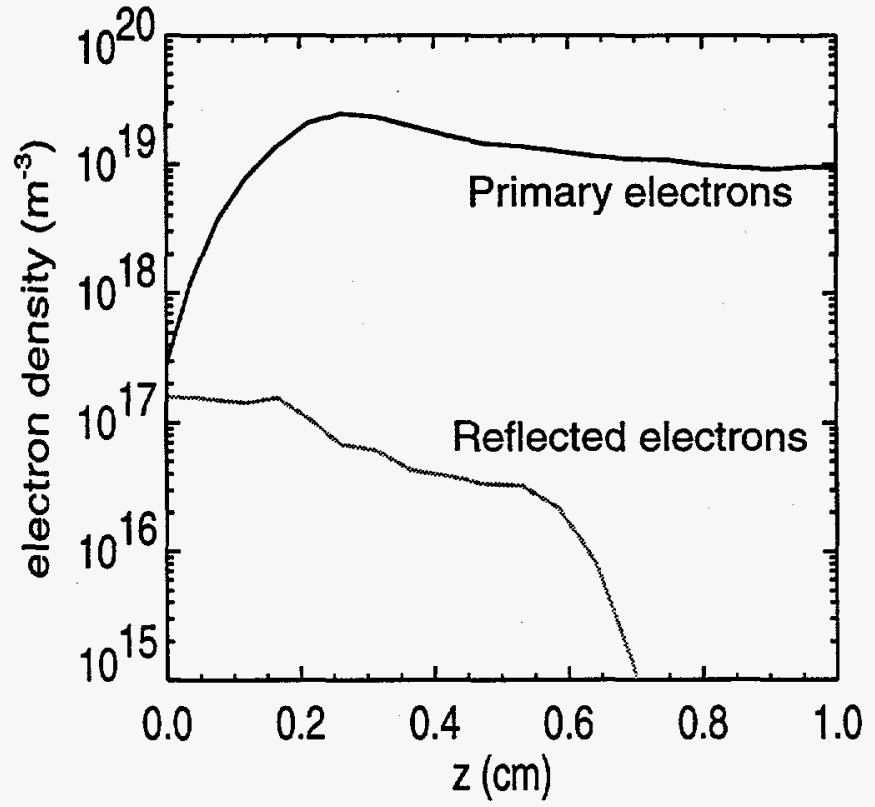

R. Vesey

Phys. Plasmas

Figure 6. 


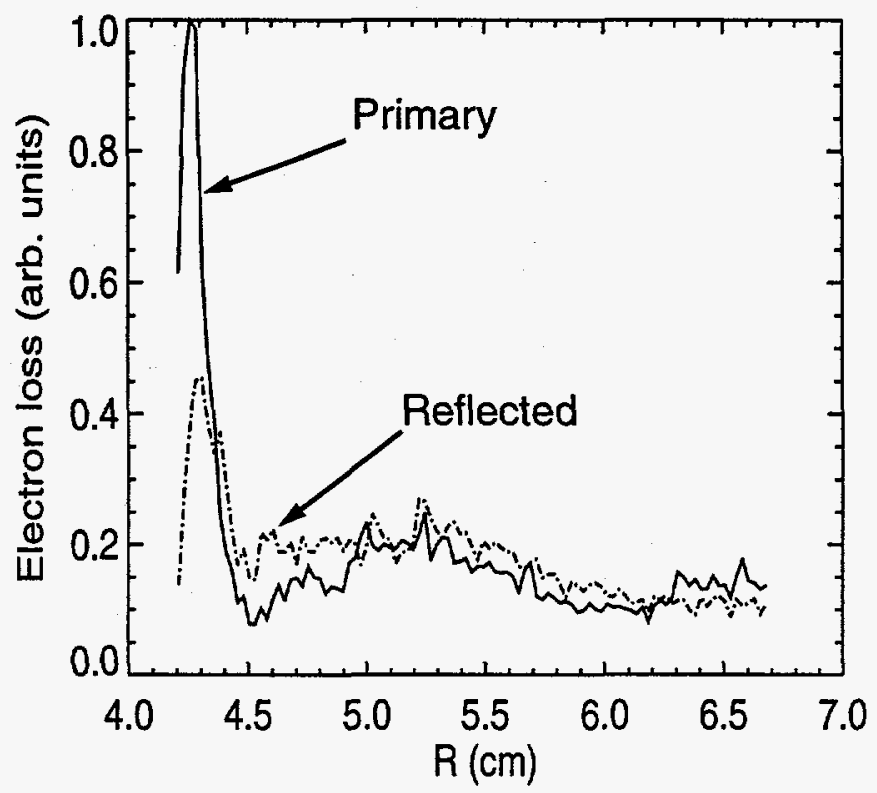

Figure 7. 


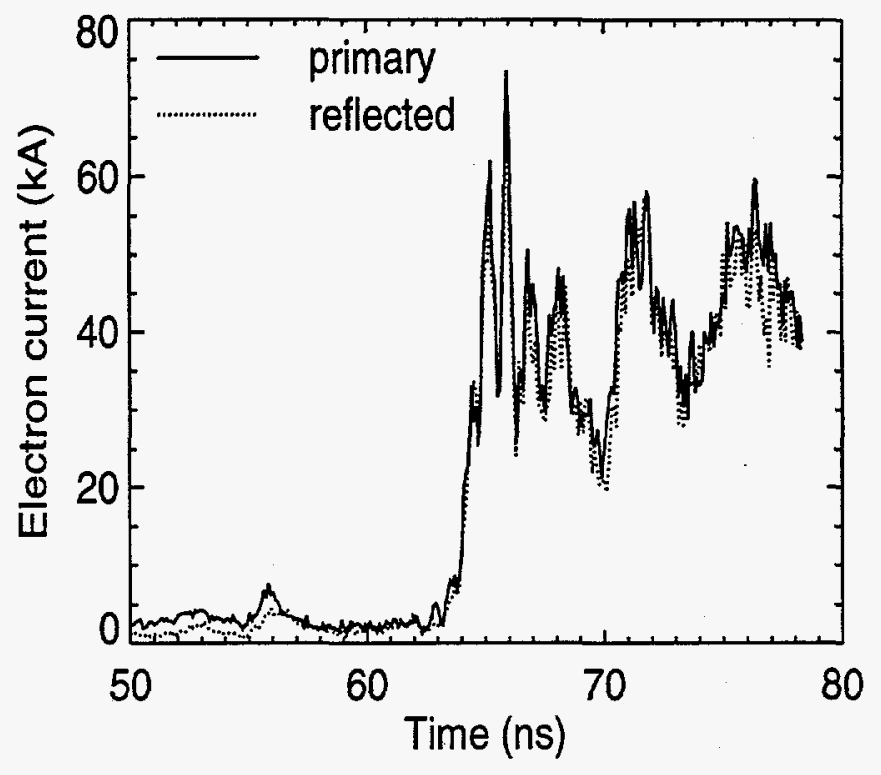

R. Vesey

Phys. Plasmas
Figure 8. 

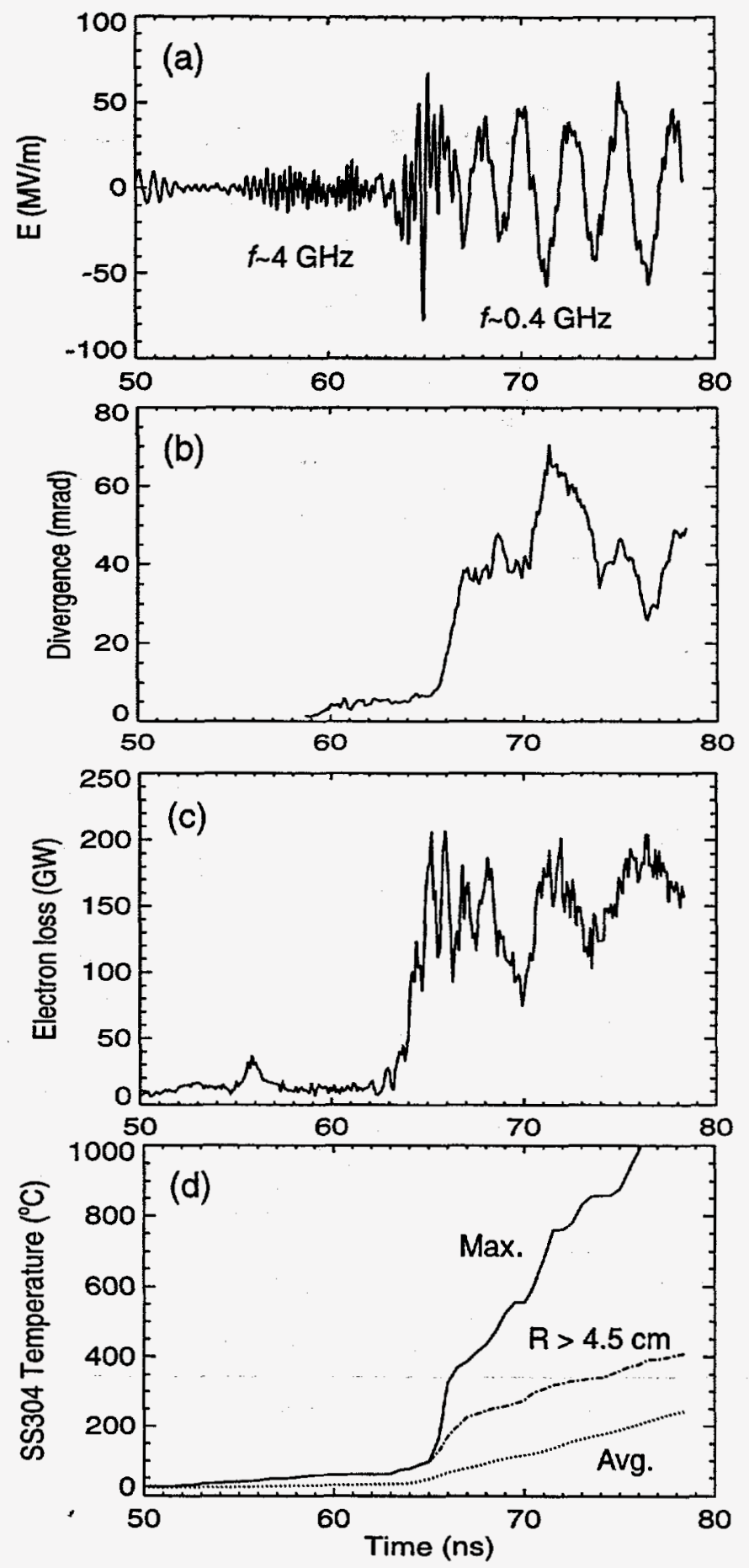

R. Vesey

Phys. Plasmas
Figure 9. 


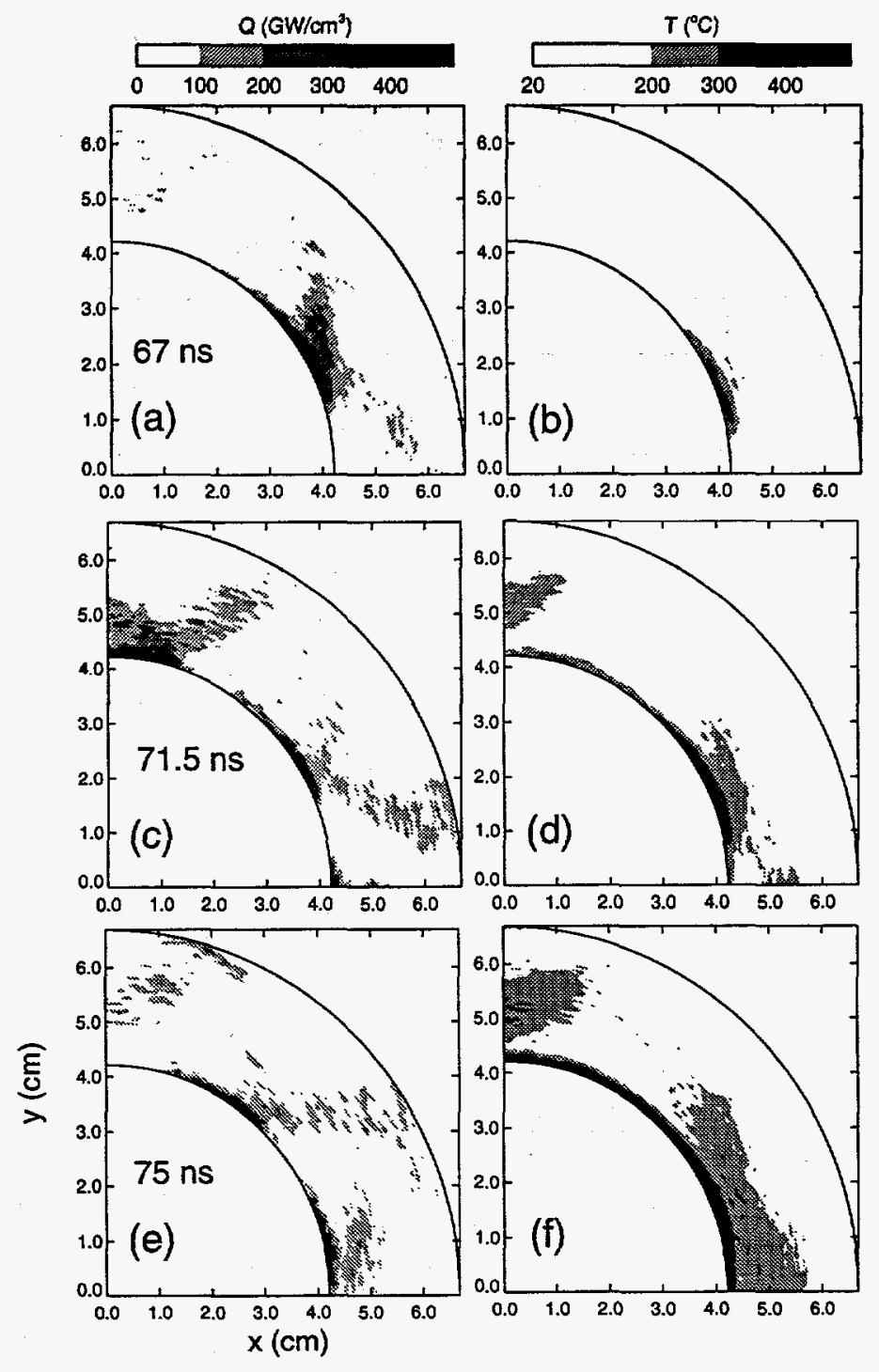




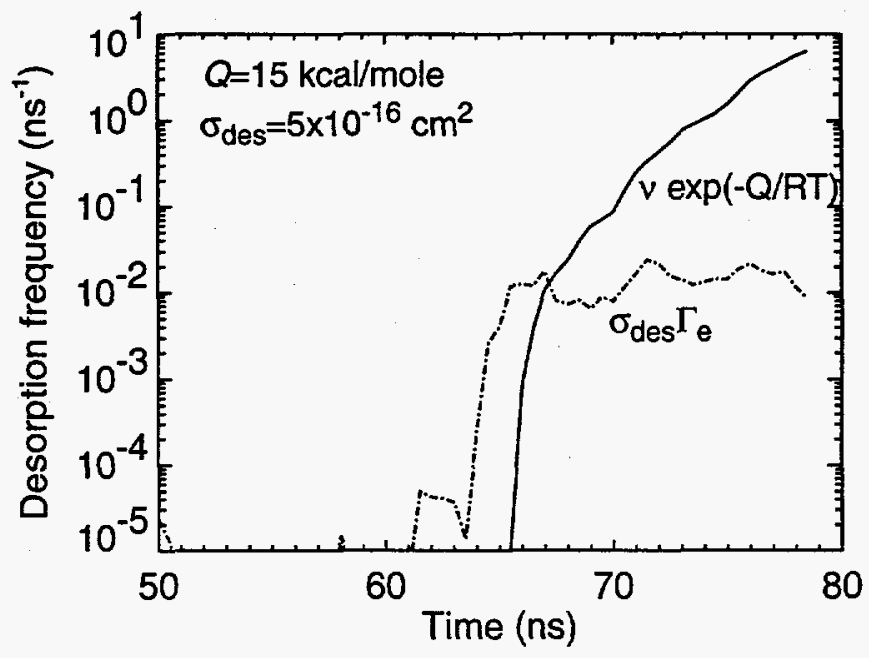

R. Vesey

Phys. Plasmas
Figure 11. 

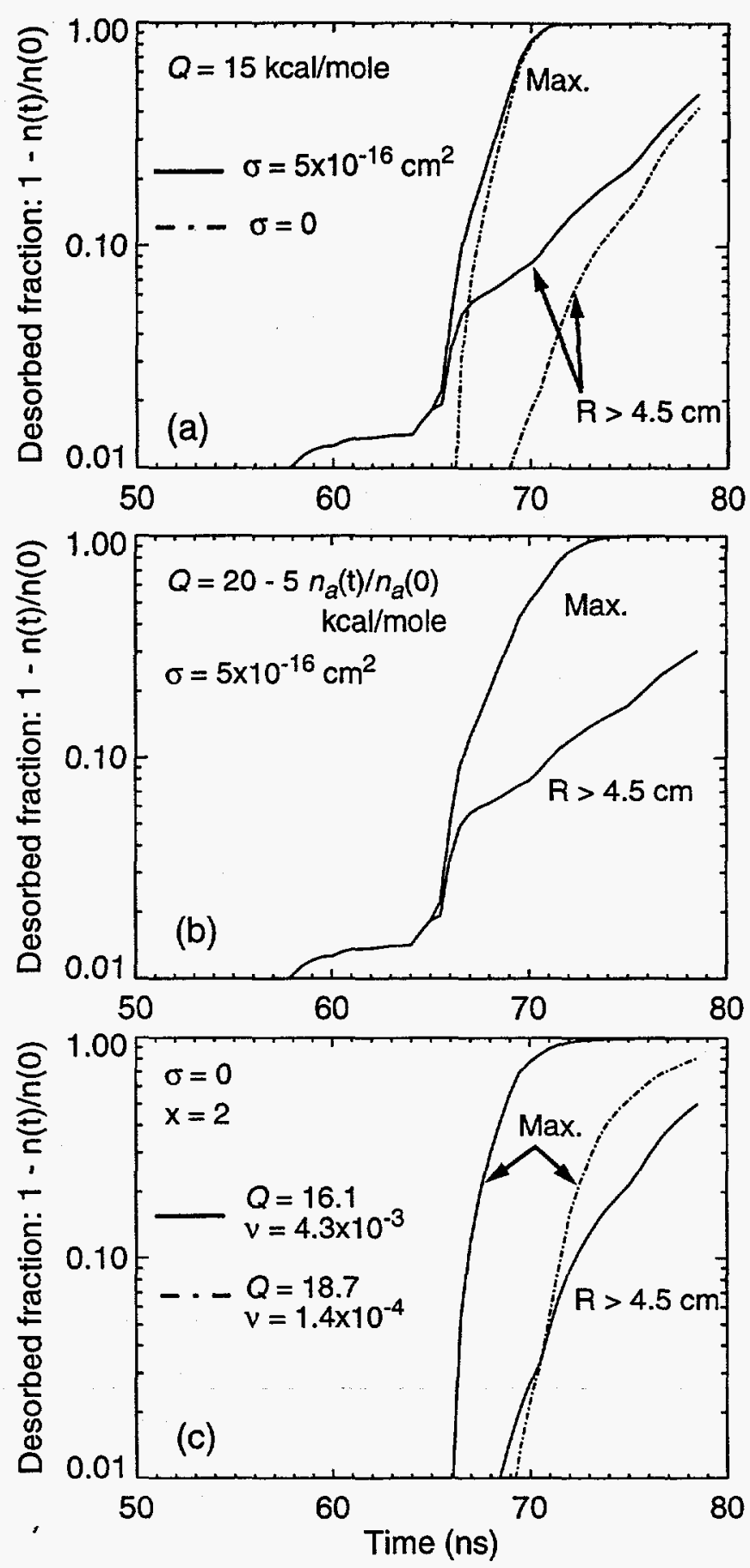

R. Vesey

Phys. Plasmas
Figure 12. 

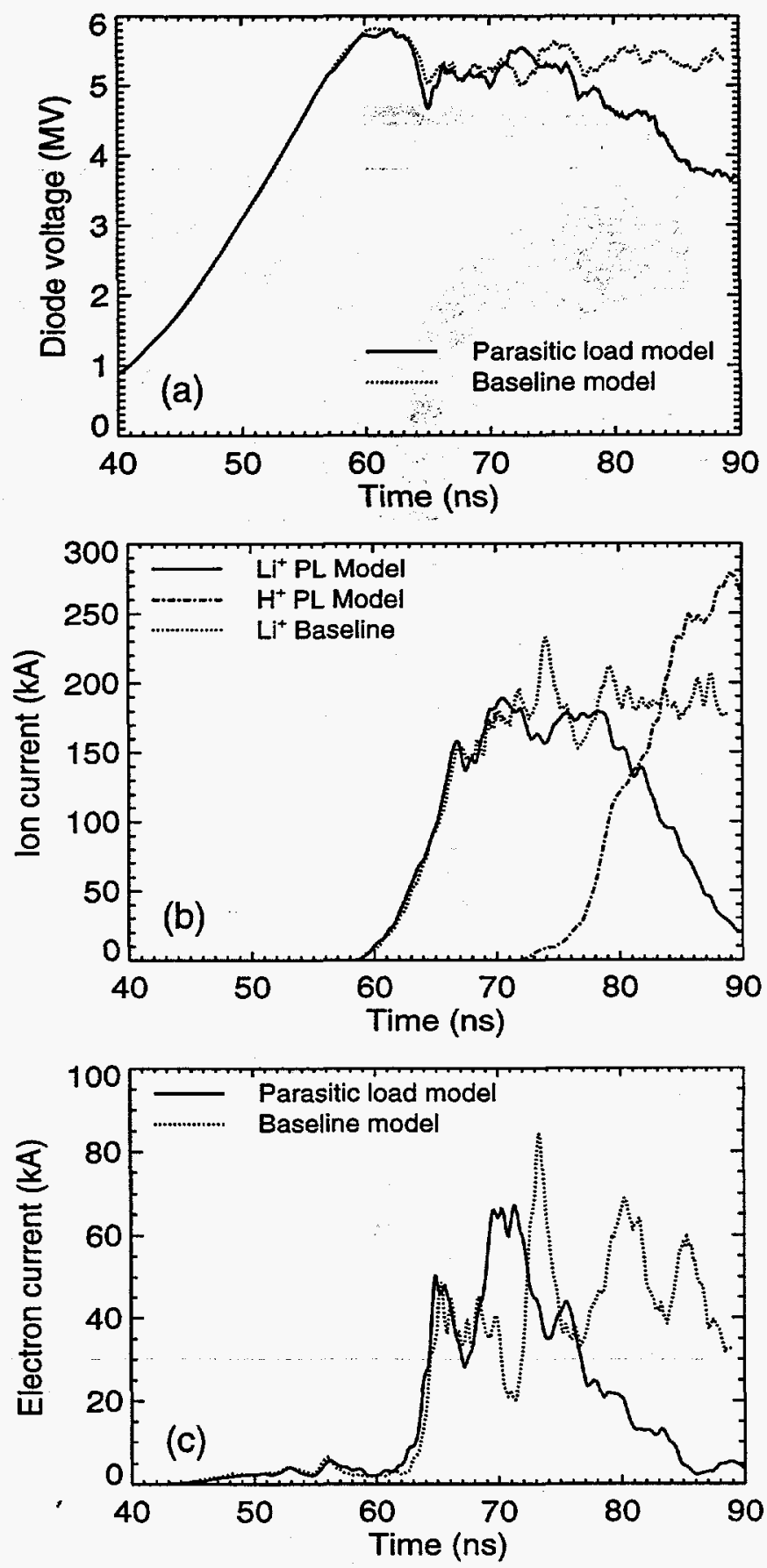

R. Vesey

Phys. Plasmas
Figure 13. 

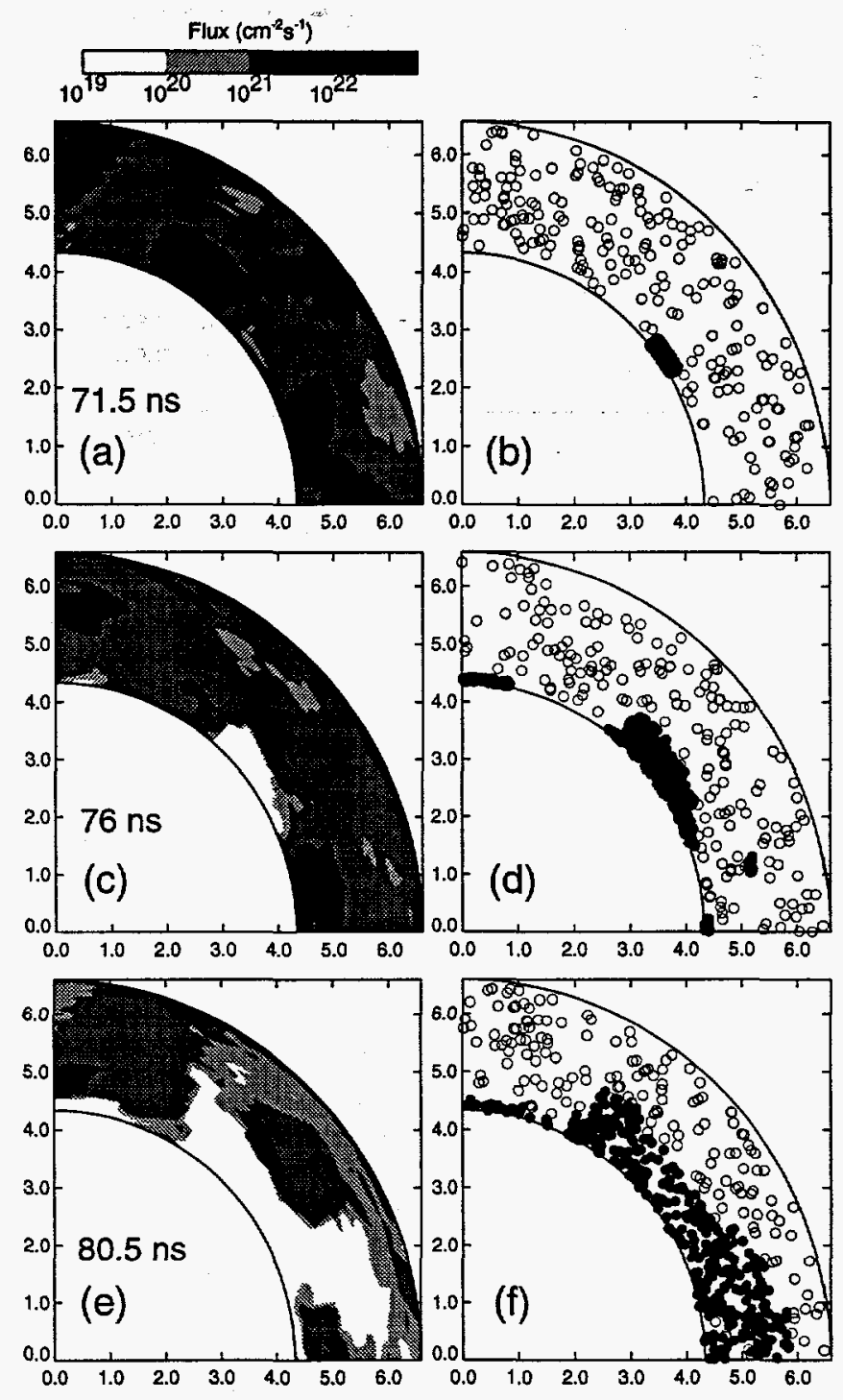

R. Vesey

Phys. Plasmas
Figure 14. 

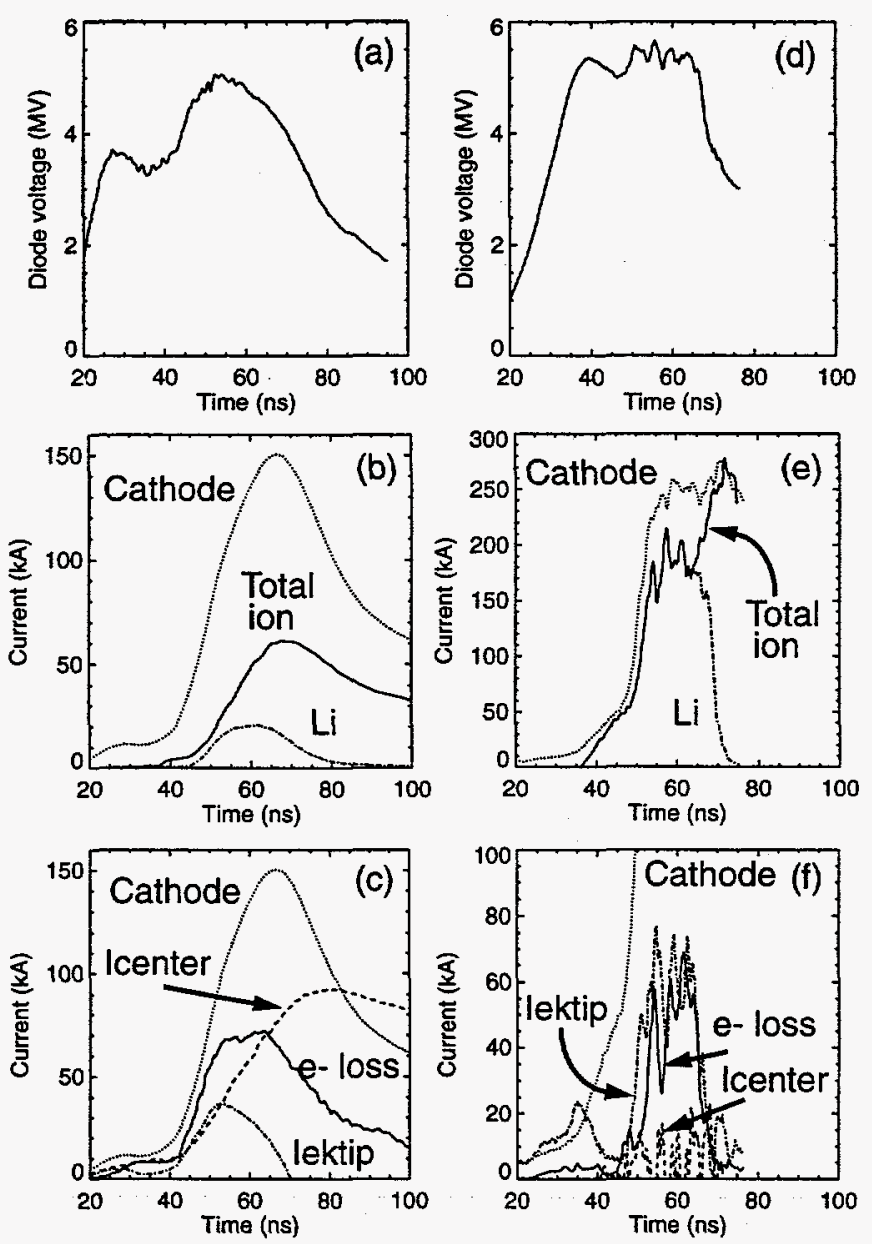

R. Vesey

Phys. Plasmas
Figure 15. 

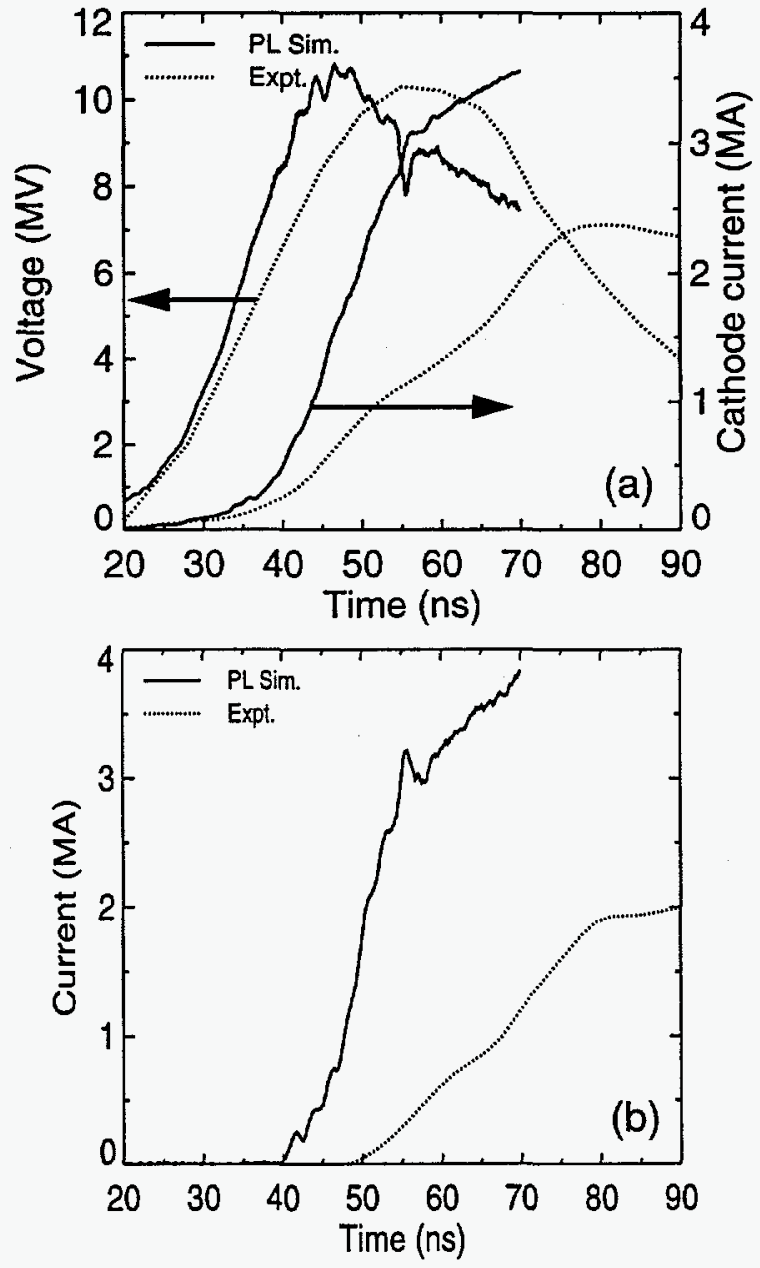

R. Vesey

Phys. Plasmas
Figure 16. 

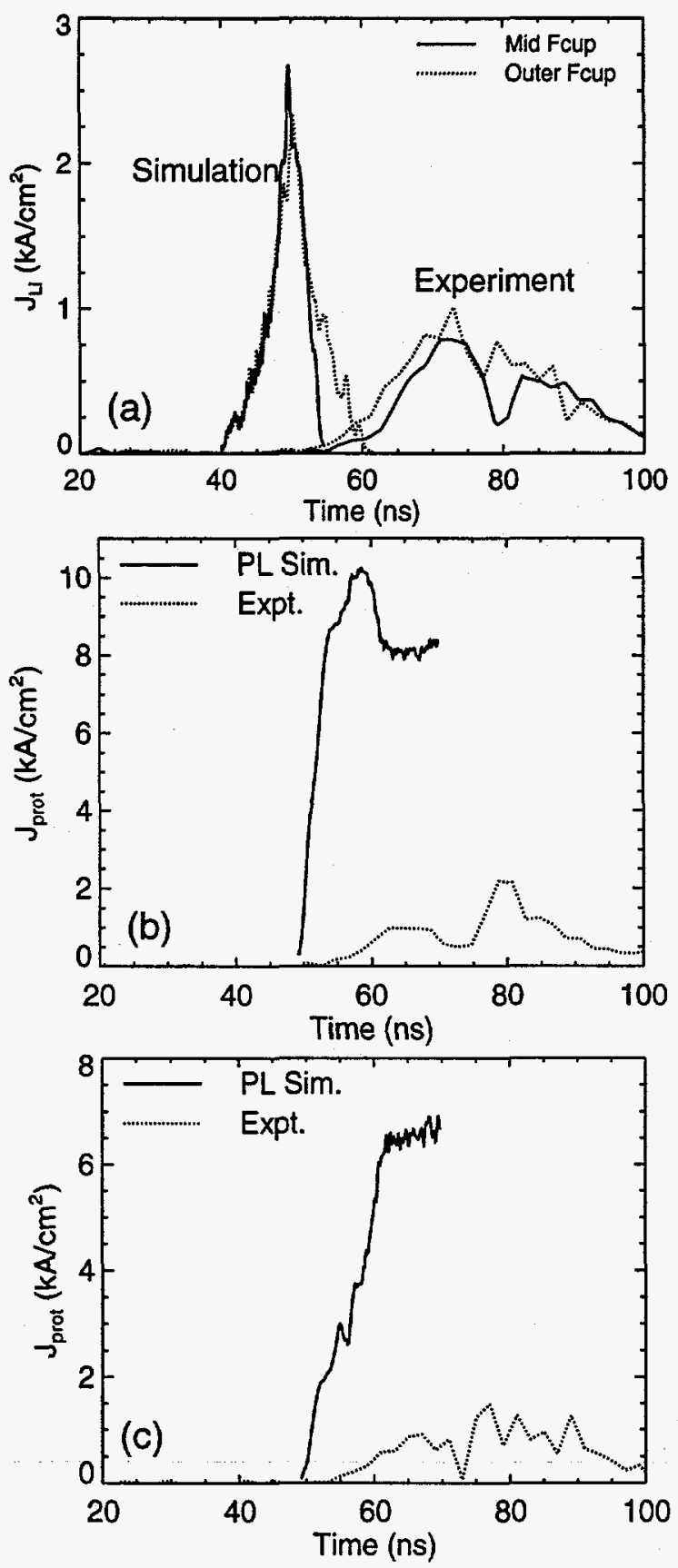

Figure 17. 


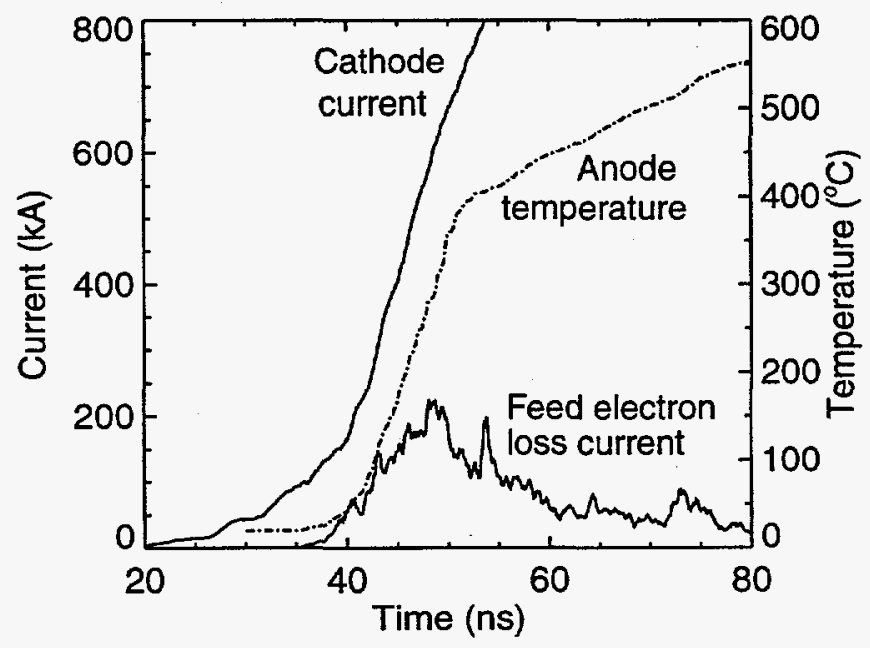

R. Vesey

Phys. Plasmas

Figure 18. 


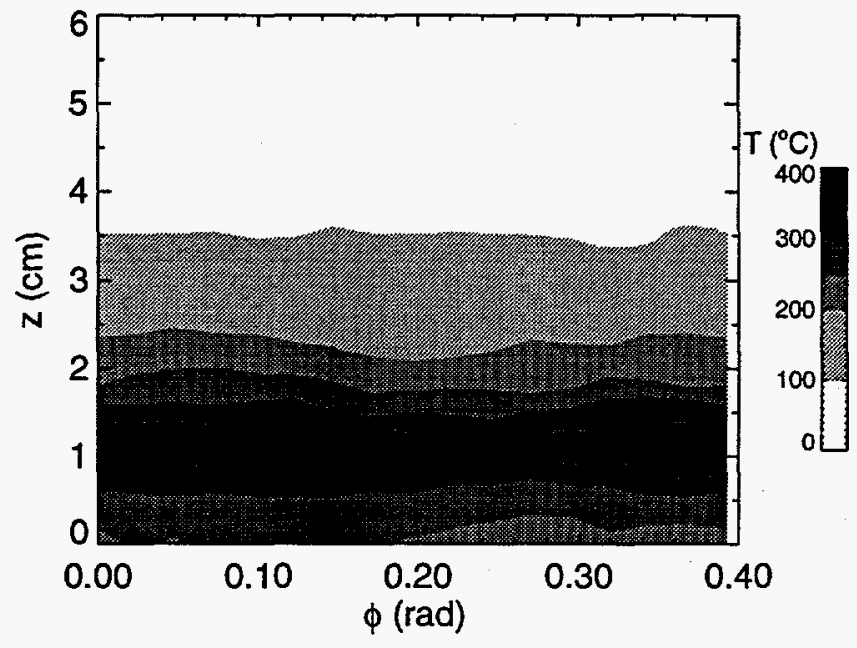

R. Vesey

Phys. Plasmas
Figure 19. 

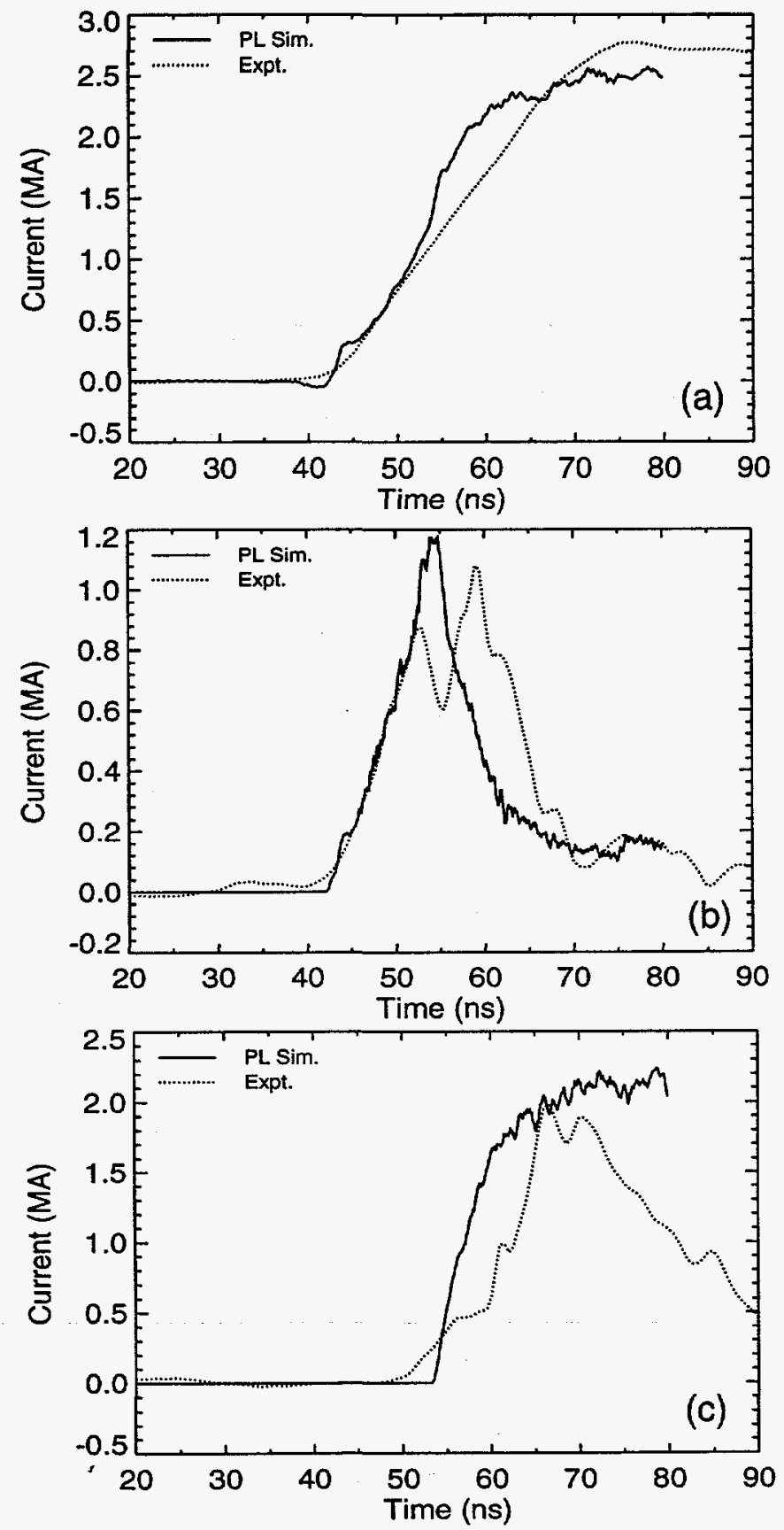

R. Vesey

Phys. Plasmas
Figure 20. 

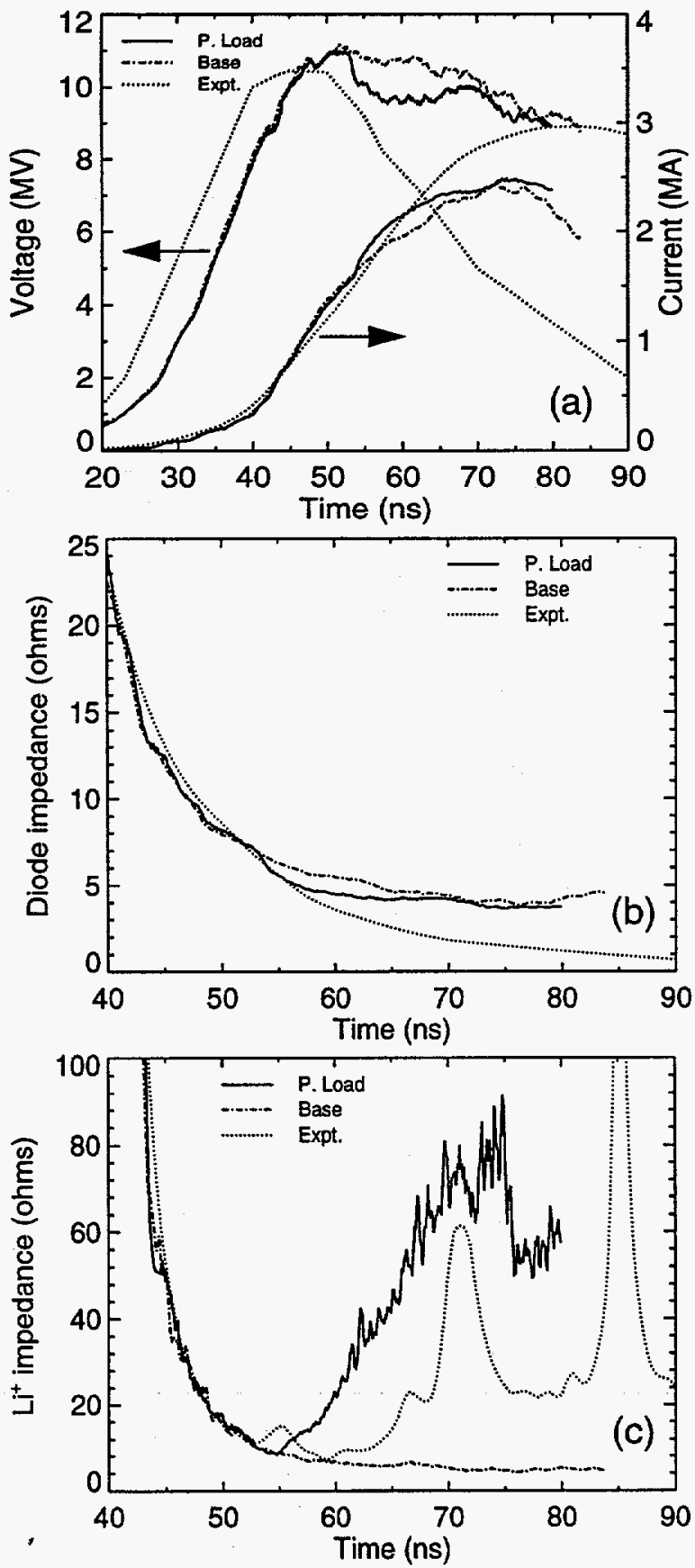

R. Vesey

Phys. Plasmas
Figure 21. 

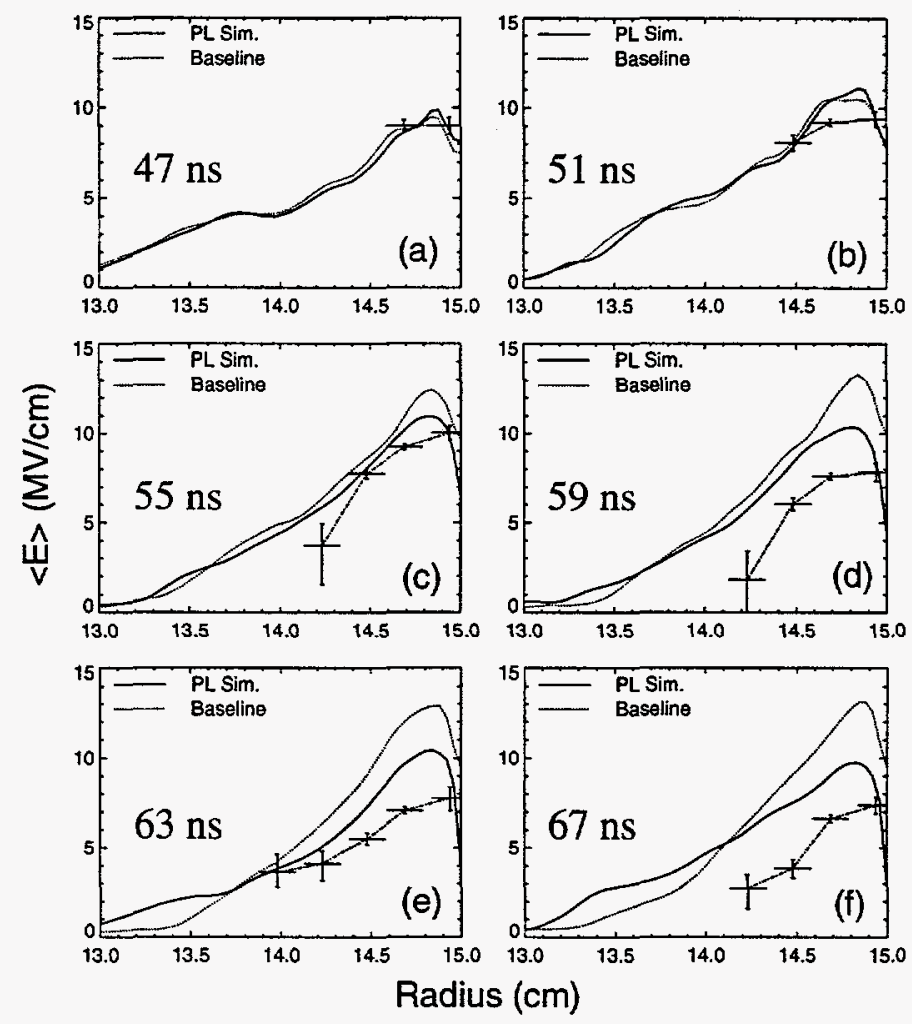

R. Vesey

Phys. Plasmas
Figure 22. 